\title{
Activation of PINK1-Parkin-Mediated \\ Mitophagy Degrades Mitochondrial Quality \\ Control Proteins in Fuchs Endothelial \\ Corneal Dystrophy
}

Takashi Miyai, ${ }^{*}$ Shivakumar Vasanth, ${ }^{*}$ Geetha Melangath, ${ }^{*}$ Neha Deshpande, ${ }^{*}$ Varun Kumar, ${ }^{*}$ Anne-Sophie Benischke, ${ }^{*}$ Yuming Chen, ${ }^{*}$ Marianne 0. Price, ${ }^{\dagger}$ Francis W. Price, $\mathrm{Jr}^{\dagger}{ }^{\star}$ and Ula V. Jurkunas*

From the Schepens Eye Research Institute, * Massachusetts Eye and Ear, Department of Opthalmology, Harvard Medical School, Boston, Massachusetts; and the Price Vision Group ${ }^{\dagger}$ Indianapolis, Indiana

\author{
Accepted for publication \\ June 11, 2019. \\ Address correspondence to Ula \\ V. Jurkunas, M.D., Schepens \\ Eye Research Institute, Massa- \\ chusetts Eye and Ear, Depart- \\ ment of Ophthalmology, \\ Harvard Medical School, 20 \\ Staniford St., Room 245, Bos- \\ ton, MA 02114. E-mail: ula \\ jurkunas@meei.harvard.edu.
}

\begin{abstract}
Corneal endothelium (CE) is a monolayer of mitochondria-rich cells, critical for maintaining corneal transparency compatible with clear vision. Fuchs endothelial corneal dystrophy (FECD) is a heterogeneous, genetically complex disorder, where oxidative stress plays a key role in the rosette formation during the degenerative loss of CE. Increased mitochondrial fragmentation along with excessive mitophagy activation has been detected in FECD; however, the mechanism of aberrant mitochondrial dynamics in CE cell loss is poorly understood. Here, the role of oxidative stress in mitophagy activation in FECD is investigated. Immunoblotting of FECD ex vivo specimens revealed an accumulation of PINK1 and phospho-Parkin (Ser65) along with loss of total Parkin and total Drp1. Similarly, modeling of rosette formation with menadione (MN), led to phospho-Parkin accumulation in fragmented mitochondria resulting in mitophagy-induced mitochondrial clearance, albeit possibly in a PINK1independent manner. Loss of PINK1, phospho-Drp1, and total Drp1 was prominent after MN-induced oxidative stress, but not after mitochondrial depolarization by carbonyl cyanide m-chlorophenyl hydrazone. Moreover, MN-induced mitophagy led to degradation of Parkin along with sequestration of Drp1 and PINK1 that was rescued by mitophagy inhibition. This study shows that in FECD, intracellular oxidative stress induces Parkin-mediated mitochondrial fragmentation where endogenous Drp1 and PINK1 are sequestered and degraded by mitophagy during degenerative loss of post-mitotic cells of ocular tissue. (Am J Pathol 2019, 189: 2061-2076; https://doi.org/10.1016/j.ajpath.2019.06.012)
\end{abstract}

Corneal endothelium (CE), the innermost layer of the cornea, is composed of morphologically distinct hexagonal monolayer of post-mitotically arrested differentiated cells with a high density of mitochondria. ${ }^{1}$ The presence of abundant mitochondria provides the source of energy for the active functioning of ionic pumps that keep the cornea in the state of deturgescence and maintain corneal transparency required for clear vision. ${ }^{2}$ Fuchs endothelial corneal dystrophy (FECD) is a genetically complex disorder, with intronic trinucleotide repeat expansions in TCF4 gene accounting for the majority of genetic associations. ${ }^{3}$ The key characteristics of FECD include accumulation of extracellular deposits called guttae with concurrent loss of CE cells causing corneal edema and blindness. FECD affects $4 \%$ of the US population and is the leading cause of corneal transplantation in the United States and worldwide. ${ }^{4-6}$ Regardless of the genetic heterogeneity, the final common

\footnotetext{
Supported by NIH/National Eye Institute grant R01EY020581 (U.V.J.), Alcon Young Investigator grant (U.V.J.), NIH core grant P30EY003790, Bausch and Lomb, Ocular Surface Research fellowship (T.M.), Japan Eye Bank Association Overseas grant (T.M.), Nakayama Foundation International exchange grant (T.M.), American Heart Association Postdoctoral Fellowship 18POST34030385 (V.K.), and Massachusetts Lions Eye Research Fund (A.S.B.)

T.M. and S.V. contributed equally to this work.

Disclosures: M.O.P. and F.W.P. are employed by Price Vision Group.
} 
mechanism of CE cell degeneration in FECD has been linked to oxidative stress-induced apoptosis. ${ }^{7-12}$

Loss of Nrf2-NAD(P)H quinone dehydrogenase (NQO1)-mediated antioxidant defense leads to oxidant-antioxidant imbalance in FECD, causing oxidative DNA damage evidenced by the colocalization of 8-hydroxy$2^{\prime}$-deoxyguanosine with mitochondria in ex vivo endothelial whole mounts. ${ }^{8}$ Specifically, decreased expression of superoxide dismutase (SOD-2) and peroxiredoxins (PRX-2, PRX-5) in FECD suggests reduced mitochondrial antioxidant capacity. ${ }^{8,13}$ Previous studies have identified decreased mitochondrial density, decreased activity of cytochrome oxidase, and a decrease in mitochondrial DNA-encoded transcripts in FECD. ${ }^{14,15}$ In their recent studies, the authors identified a stark reduction in mitochondrial membrane potential $(\Delta \psi \mathrm{m})$; increased mitochondrial DNA damage, and fragmentation of mitochondria ${ }^{16}$; and downstream induction of mitophagy supported by the increased levels of LC3-II autophagy marker in FECD specimens. ${ }^{1}$ These observations were further corroborated by the presence of degenerated mitochondria within autophagic vacuoles by ultrastructural analysis of ex vivo FECD specimens indicating constitutive activation of mitophagy. However, the mechanism of purportedly aberrant mitochondrial dynamics in FECD has not been investigated. ${ }^{16}$

The healthy state of mitochondria is determined by three key pathways essential for mitochondrial quality control, namely, fission, fusion, and mitophagy (the selective degradation of depolarized mitochondria through autophagy). ${ }^{17}$ Dynamin-related protein 1 (Drp1), the key regulator of fission, forms an oligomeric ring-like structure and leads to the division of mitochondria. ${ }^{18}$ Phosphorylation of Ser616 on Drp1 due to increase in reactive oxygen species (ROS) has been shown to promote the fission pathway ${ }^{19}$ required for the equal distribution of mitochondria to daughter cells in mitosis. ${ }^{20}$ Phosphorylation of Ser637 on Drp1 by protein kinase A impairs mitochondrial fission due to the inhibition of its GTPase activity and is associated with reduced fission. ${ }^{21,22}$ Drp1 is also regulated by calcineurin, a cytosolic phosphatase that is activated by rise in intracellular $\mathrm{Ca}^{2+}$, which dephosphorylates Ser637 and results in the translocation of Drp1 to mitochondria resulting in the fragmentation of depolarized mitochondria. ${ }^{23}$ Mitochondrial fusion, on the other hand, involves fusion of both the inner and outer membrane and is mediated by mitofusins ( 1 and 2), which have been shown to be deficient in FECD. ${ }^{1,24}$ When repetitive cycles of mitochondrial fusion and fission are unable to rescue the extensive mitochondrial damage, mitophagy flux increases in the cells to aid the sequestration of unsalvageable mitochondrial population. Loss of $\Delta \psi \mathrm{m}$, the major trigger for mitophagy, results in the stabilization of PTEN-induced putative kinase 1 (PINK1) ${ }^{25}$ in the outer mitochondrial membrane, which is essential for recruiting the cytosolic E3 ubiquitin ligase Parkin ${ }^{26}$ and ubiquitin by phosphorylating both proteins at Ser65. ${ }^{27-29}$ Although PINK1 directly phosphorylates Parkin at Ser65, the simultaneous phosphorylation of ubiquitin at Ser65 is necessary for the complete activation of Parkin and its translocation to mitochondria. ${ }^{30,31}$ Parkin further ubiquitinates its substrates on the outer mitochondrial membrane, which are subjected to proteasomal degradation. The ubquitination primes the mitochondria for the recruitment of phagophores, ${ }^{32}$ thereby clearing the remaining bulk of mitochondrion through the autophagosomal pathway. ${ }^{33-35}$

The majority of findings on the prevailing mechanism of mitophagy are based on the engineered overexpression of proteins and induction of mitochondrial depolarization by carbonyl cyanide $m$-chlorophenyl hydrazine (CCCP). ${ }^{28,31,36}$ However, there is a scarcity of evidence on the mitophagy activation in response to oxidative stress relative to endogenous mitochondrial qualitycontrol protein activation. Menadione (2-methyl-1,4naphthoquinone) (MN), a quinone that generates mitochondrial superoxide ${ }^{16}$ and an unstable semiquinone radical, ${ }^{37}$ induces extensive oxidative mitochondrial DNA and nuclear DNA damage leading to mitochondrial fragmentation ${ }^{16}$ and dysfunction, culminating in apoptosis, during the rosette formation in FECD. Morphologically, one of the key characteristics of FECD is the disruption of the $\mathrm{CE}$ monolayer by rosette formation, where $\mathrm{CE}$ cell loss forms a ring or rosette around the bases of extracellular matrix deposits or guttae. ${ }^{1,16,38}$ Therefore, in this study we investigated the mechanism by which intracellular oxidative stress, seen specifically in FECD activates mitophagy during the rosette formation, which is a prominent feature of degenerating CE cells. ${ }^{14,39,40}$ The role of the mitophagy signaling cascade in human $\mathrm{CE}$ cells (HCEnCs) retrieved from FECD patients was first studied. The in vitro modeling of the pathognomonic rosette formation with $\mathrm{MN}$ in $\mathrm{CE}$ cells recapitulated the differences in the phospho-Parkin-mediated mitophagy pathway intermediates observed in ex vivo specimens and provided new evidence on the intrinsic mitochondrial quality control changes in response to oxidative stress. MN induced Parkin recruitment into fragmented mitochondria, creating a model system for the visualization of Parkin-dependent mitochondrial dynamics due to ROS. Parkin translocation to mitochondria triggered protein ubiquitination and proteasomal degradation, and activated excessive mitochondrial loss via mitophagy in FECD. In addition, Parkin mediated the sequestration of Drp1 and PINK1, furthering our understanding of the role of oxidative stress on endogenous protein clearance in the post-mitotic cells of ocular tissue.

\section{Materials and Methods}

\section{Human Tissue}

Normal human corneas were obtained from Northeast Pennsylvania Lions Eye Bank (Bethlehem, PA), Eversight (Ann Arbor, MI), Lions VisionGift (Portland, OR), SightLife (Seattle, WA), and Tissue Banks International 
(Baltimore, MD). Donor corneas were recovered and preserved in Optisol-GS (Bausch \& Lomb, Rochester, NY) within 24 hours of death and stored at refrigerated temperature $\left(4^{\circ} \mathrm{C}\right)$ until use. CE tissue from FECD cases were isolated by endothelial keratoplasty performed at Price Vision Group (Indianapolis, IN), Massachusetts Eye and Ear Infirmary (Boston, MA), and Ophthalmic Consultants of Boston (Waltham, MA). Briefly, FECD surgical explants were obtained by stripping the central $7 \mathrm{~mm}$ to $8 \mathrm{~mm}$ of Descemet's membrane and endothelium from the recipient's posterior cornea ${ }^{41}$ and stored at refrigerated temperature in Optisol up to a maximum of 7 days before use. The current study was performed according to the tenets of the Declaration of Helsinki and approved by the Massachusetts Eye and Ear Institutional Review Board. Written and informed consent was obtained from patients before endothelial keratoplasty. The age, sex, and the duration of storage of all of the normal donor and FECD patient tissues used in this study are listed in Supplemental Table S1, based on the parameters used in the authors' previous study. ${ }^{13}$ The CE densities of the normal corneas chosen for this study are in the range of 1800 to 3200 cells $/ \mathrm{mm}^{2}$.

\section{Human CE Cell Culture}

Normal CE cell line (HCEnC-21T) was previously generated in the authors' laboratory using retrovirus transfection containing pBABE-puro-hTERT. ${ }^{42} \mathrm{CE}$ cell lines derived from normal CE (HCECi) and FECD patient's CE (FECDi) were gifts from Dr. May Griffith (Ottawa Hospital Research Institute, Ottawa, ON) and Dr. Rajiv Mohan (University of Missouri Health System, Columbia, MO), respectively. ${ }^{43,44}$ Cells were cultured in Chen's medium containing OptiMEM I (Thermo Fisher Scientific, Waltham, MA), 8\% fetal bovine serum (HyClone, Rockford, IL), $5 \mathrm{ng} / \mathrm{mL}$ epidermal growth factor (Millipore, Billerica, MA), $100 \mathrm{mg} / \mathrm{mL}$ bovine pituitary extract (Alfa Aesar by Thermo Fisher Scientific, Haverhill, MA), $200 \mathrm{mg} / \mathrm{L}$ calcium chloride (Sigma-Aldrich, St. Louis, MO), $0.08 \%$ chondroitin sulfate (Sigma-Aldrich), $50 \mathrm{mg} / \mathrm{mL}$ gentamicin (Thermo Fisher Scientific), and 1:100 diluted antibiotic/antimycotic solution (Sigma-Aldrich). The CE cells were subcultured using $0.05 \%$ Trypsin-EDTA (Invitrogen) for 2 minutes at $37^{\circ} \mathrm{C}$. For the $\mathrm{MN}$ treatment, 1.2 million HCEnCs were plated in Chen's medium for 24 hours followed by treatment with $50 \mu \mathrm{mol} / \mathrm{L} \mathrm{MN}$ or $20 \mu \mathrm{mol} /$ L CCCP in Dulbecco's modified Eagle's medium (Thermo Fisher Scientific). Cells were later harvested at 4, 8, and 20 hours after treatment for Western blot analysis. Cells were pretreated with $1 \mu \mathrm{mol} / \mathrm{L}$ epoxomicin 30 minutes before $\mathrm{MN}$ or CCCP treatment, whereas $10 \mathrm{nmol} / \mathrm{L}$ of bafilomycin $\mathrm{A} 1$ was added along with $\mathrm{MN}$ or CCCP treatment.

\section{Transfection}

HCEnC-21T cells (0.6 million) were transfected in suspension with $0.4 \mu \mathrm{g}$ of YFP-Parkin expressing plasmid (23955;
Addgene, Cambridge, MA) with $0.8 \mu \mathrm{L}$ Lipofectamine 2000 (Thermo Fisher Scientific) in a total of $80 \mu \mathrm{L}$ of OptiMEM (Thermo Fisher Scientific) and incubated for 20 minutes. Cells were treated with $20 \mu \mathrm{mol} / \mathrm{L}$ CCCP (C2759; SigmaAldrich) or 10,25 , or $50 \mu \mathrm{mol} / \mathrm{L}$ menadione sodium bisulfite (M2518; Sigma-Aldrich) 30 hours after transfection and further taken for immunofluorescence staining.

\section{Immunocytochemistry}

Immunofluorescence staining and Western blot analysis were performed as described previously. ${ }^{16}$ For immunofluorescence, HCEnC-21T cells were seeded in a 12-well plate coated with fibronectin and treated with increasing doses of MN (10, 25, and $50 \mu \mathrm{mol} / \mathrm{L} \mathrm{MN})$ for 20 hours. The cells were fixed with $4 \%$ paraformaldehyde for 20 minutes at room temperature. After washing with phosphate-buffered saline three times, they were permeabilized with $0.15 \%$ Triton X-100 for 15 minutes and blocked with $10 \%$ bovine serum albumin. Primary antibodies for cytochrome $c$ antibody (BD 556432; Becton, Dickinson and Company, Franklin Lakes, NJ) were incubated at $4^{\circ} \mathrm{C}$ overnight followed by secondary Alexa 546-conjugated antibody (Invitrogen; Thermo Fisher Scientific) for 1 to 2 hours at room temperature. Nuclei were stained with DAPI (Vectashield; H1000) and imaged using confocal microscopy (Carl Zeiss, Oberkochen, Germany) at different magnifications $(40 \times, 63 \times$, zoom 1.5 , and zoom 4$)$.

\section{Western Blot Analysis}

Whole-cell lysates were prepared by lysing MN-, bafilomycin-, and CCCP-treated cells in radioimmunoprecipitation assay buffer containing HALT protease and phosphatase inhibitor cocktail $(100 \times)$ (78440; Thermo Fisher Scientific), and the protein concentration was estimated using BCA protein assay (Thermo Fisher Scientific). Proteins were separated by SDS-PAGE on a $12 \%$ Bis-Tris gel and transferred onto a polyvinylidene difluoride membrane (MilliporeSigma, Burlington, MA) followed by 1 hour of blocking in 5\% nonfat milk in phosphate-buffered saline $0.1 \%$ Tween20. Primary antibodies phospho-Drp1 (Ser637) (PA5-37534; Thermo Fisher Scientific), phospho-Drp1 (Ser616) (3455; Cell Signaling Technology, Danvers, MA), Total Drp1 (BD 611112; Becton, Dickinson and Company), Parkin (ab15954; Abcam, Cambridge, MA), phospho-Parkin (Ser65) (ab154995; Abcam), PINK1 (BC100-494; Novus Biologicals, Centennial, CO), Actin (A1978; SigmaAldrich), LC3A/B (4108; Cell Signaling Technology), voltage-dependent anion channel (VDAC) (ab18988; Abcam), or Ubiquitin (3936; Cell Signaling Technology) were incubated with the polyvinylidene difluoride membrane overnight at $4^{\circ} \mathrm{C}$. Relative protein levels were compared by quantifying band intensities from scanned X-ray films using ImageJ software version 1.47 (NIH, Bethesda, MD; http://imagej.nih.gov/ij). 


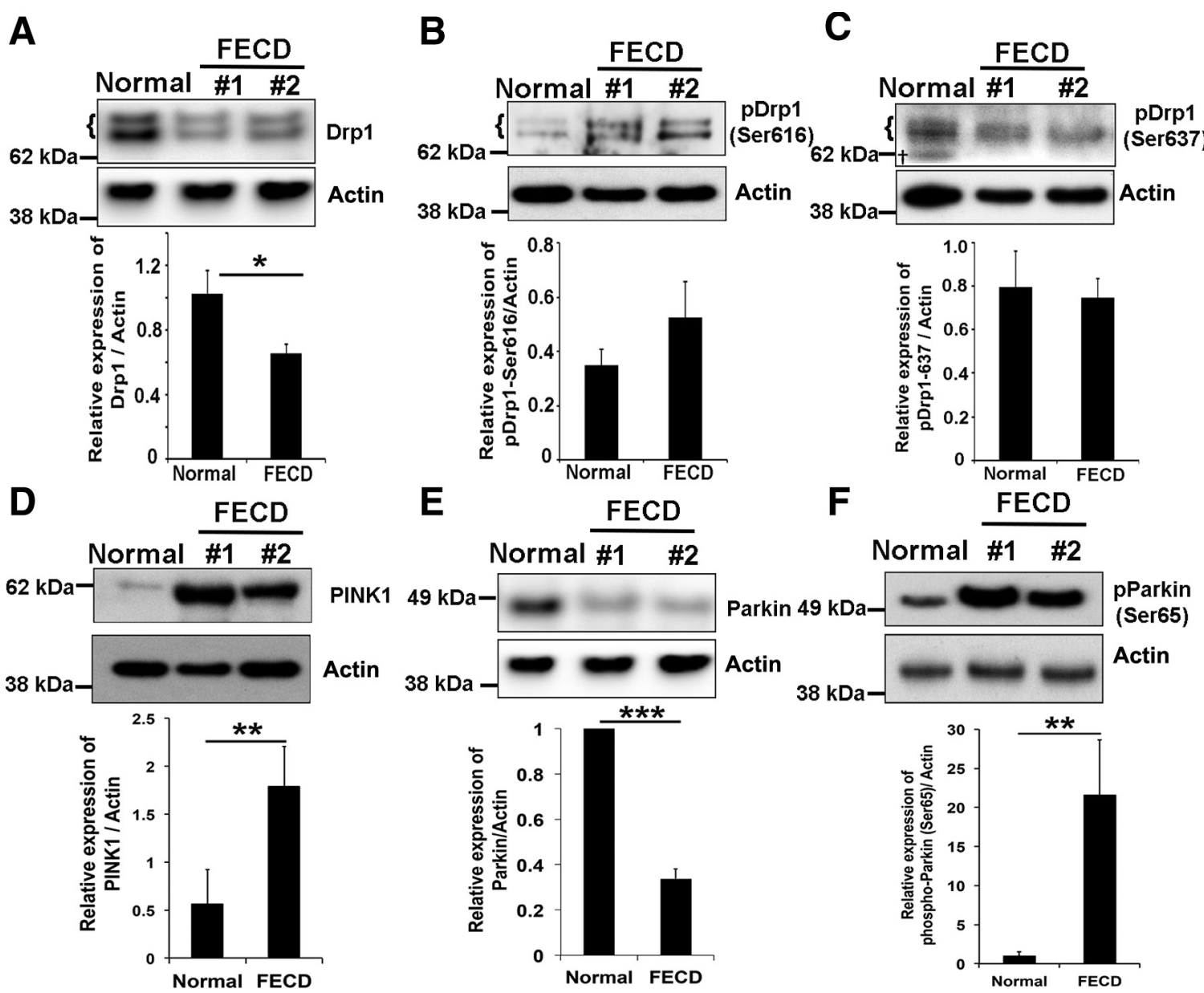

Figure 1 Fuchs endothelial corneal dystrophy (FECD) specimens reveal decreased Drp1, accumulation of PINK1, and activation of Parkin. A-C: Western blot analysis of total Drp1 (A), phospho-Drp1 (Ser616) (B), and phospho-Drp1 (Ser637) (C) in the whole-cell lysates of ex vivo specimens. $\beta$-actin is used as the normalizing control. Brackets indicate the doublet band observed for Drp1 and its phosphorylated forms. The nonspecific band observed in phosho-Drp1 (Ser37) Western blots is denoted by the dagger symbol. D: Western blot on the whole-cell lysates of normal and FECD specimens for PINK1 after normalization with $\beta$-actin. $\mathbf{E}$ and $\mathbf{F}$ : Immunoblotting analysis of total Parkin (E), and phospho-Parkin (Ser65) (F) in the whole-cell lysates. Data are expressed as means \pm SEM. $n=7$ normal specimens (A); $n=23 \mathrm{FECD}$ specimens (A); $n=3$ normal specimens (B, D-F); $n=8 \mathrm{FECD}$ specimens (B and C); $n=5$ normal specimens $(\mathbf{C}) ; n=4$ FECD specimens (D and $\mathbf{E}) ; n=6$ FECD specimens $(\mathbf{F})$. ${ }^{*} P<0.05,{ }^{*} P<0.01$, and ${ }^{* * *} P<0.001$ (two tailed $t$-test).

Human Descemet's membrane with CE cells were lyzed in protein extraction buffer containing ER3 (Bio-Rad Laboratories, Hercules, CA), $1 \mu \mathrm{mol} / \mathrm{L}$ tributyl phosphine (BioRad Laboratories), and Halt Protease and Phosphatase inhibitor Cocktail $(100 \times)(78440$; Thermo Fisher Scientific). Specimens were centrifuged at $57,000 \times g$ for 1 hour at $21^{\circ} \mathrm{C}$ (Optima TLX ultracentrifuge; Beckman Coulter, Danvers, MA).

\section{Mitochondrial Fractionation}

For assessing the mitochondrial levels of phospho-Parkin and LC3-II in MN-treated HCEnC-21T cells, mitochondria were purified using BioVision Mitochondria/Cytosol Fractionation Kit (Cat no-K256-25; BioVision, Milpitas, CA) according to the manufacturer's instructions. From HCECi and FECDi lines, intact mitochondria were isolated with anti-TOM22 antibody-bound magnetic beads using the Mitochondrial isolation kit (Miltenyi Biotec, San Diego, CA) according to the manufacturer's instructions. ${ }^{1}$ Briefly, cells were lyzed using lysis buffer followed by homogenization using $26-\mathrm{G}$ needle and $1-\mathrm{mL}$ syringe that mechanically disrupt the cell membrane. This procedure allows the isolation of intact vital entire mitochondria from human cells and tissues. The mitochondria were magnetically labeled with anti-TOM22 (translocase of the outer mitochondrial membrane 22) antibody microbeads. The labeled cell lysate was loaded onto a column and placed in a magnetic field separator. The magnetically labeled mitochondria were retained in the column during washing. Subsequently, the magnet was removed from the column followed by elution of mitochondria. The isolated mitochondria were later taken for Western blot analysis as described in the previous section.

\section{Quantification of Mitochondrial Fragmentation}

Cells with only fragmented mitochondria (without tubular mitochondria) were counted from a minimum of 300 cells 
from at least 10 randomly picked fields by two independent researchers (T.M. and S.V.) in a blinded manner. To test Parkin translocation induced either by MN or CCCP, cells expressing YFP-Parkin cells (total, N > 300 cells) were classified into two groups: YFP-positive cells with normal mitochondria (A) or YFP-positive cells colocalizing with fragmented mitochondria (B). Percent YFP-positive cells colocalizing with fragmented mitochondria $(\mathrm{B} / \mathrm{N})$ are represented as bar graphs.

\section{Real-Time PCR}

Real-time PCR to test the gene expression profiles of Parkin and Drp1 from total RNA from normal and FECD specimens was performed as described previously. ${ }^{1}$ Total RNA was extracted from human corneal specimens (five FECD and three normal corneas) with RNeasy micro kit (Qiagen, Hilden, Germany). Thirty nanograms of RNA were used for cDNA synthesis (iScript cDNA synthesis kit; Bio-Rad Laboratories). Relative expression of Parkin (Hs01038322_m1) and Drp1 (Hs01552605_m1) were obtained by normalizing with B2M (cat no: 4333766F) amplified using TaqMan primers (Applied Biosystems; Thermo Fisher Scientific) and Probe Fast Master Mix (Kapa Biosystems, Wilmington, MA). Triplicate real-time PCR reactions were run on an ABI StepOne Plus qPCR instrument (Applied Biosystems; Thermo Fisher Scientific). B2M was used for normalization and to calculate the relative expression.

\section{Statistical Analysis}

Two-tailed unpaired $t$-test, one way analysis of variance with post-hoc Tukey HSD (honestly significant difference) test, or two-way analysis of variance with post-hoc Tukey HSD test was used in the study as mentioned in figure legends.

\section{Results}

\section{Activation of PINK1-Parkin in the CE of FECD Patients}

The authors recently identified increased mitochondrial DNA and nuclear DNA damage, and increased mitochondrial fragmentation in FECD. ${ }^{16}$ Previous studies detected loss of mitochondrial fusion protein (Mfn2) and an increase in LC3-II, the phosphatidylethanolamine conjugated form of LC3-I that is targeted to autophagosomal membrane, along with mitophagy activation in FECD. ${ }^{1}$ Because mitochondrial fragmentation is a prominent feature of FECD cells, and fission is considered a prerequisite for activation of mitophagy, the activation of proteins involved in mitochondrial quality control (fission and mitophagy) in the post-surgical FECD specimens compared with normal CE cells was investigated. The representative Western blots for each of the proteins tested in the whole-cell lysates of multiple normal and FECD specimens are presented in
Figure 1. The activation of the essential fission protein, Drp1, and its two prominent phosphorylated forms, Ser616 and Ser637, known to promote and to be involved in the inhibition of fission, was first analyzed. ${ }^{19-21,45}$ A 2.5 -fold reduction of total Drp1 levels in the whole-cell lysates of FECD specimens $(n=23)$ as compared with normal controls was seen $(n=7, P=0.04)$, whereas the changes in phospho-Drp1(Ser616) and phospho-Drp1(Ser637) were not statistically significant in FECD specimens $(n=8)$ compared with controls $[n=3$ (Ser616), $n=5$ (Ser637)] (Figure 1, A-C). Doublet bands observed in the Western blots of Drp1 and its phosphorylated forms represent the various splice variants previously reported for Drp $1 .{ }^{46}$ The full blot for phospho-Drp1 (Ser637) is shown in the Supplemental Figure S1A. The reduction in the total Drp1 levels prompted the examination of the role of PINK1Parkin axis, because PINK1-recruited Parkin is known to ubiquitinate Drp1 and target it for degradation. ${ }^{47}$ It has been shown that during mitophagy, PINK1 phosphorylates and activates Parkin by recruiting it to the fragmented mitochondria. ${ }^{1}$ Next, it was determined whether PINK1-Parkin axis is involved during mitophagy activation in FECD. A 3.2-fold increase in full-length PINK1 in FECD specimens $(P=0.0077)$ (Figure 1D) (normal $n=3$, FECD $n=4)$ was observed, indicating the heightened recruitment of PINK1 on the outer mitochondrial membrane. Moreover, a $66 \%$ reduction in total Parkin levels $(P=0.0011)$ (Figure 1E) (normal $n=3$, FECD $n=4$ ) and an approximately 20 -fold increase in phospho-Parkin (Ser65) were observed in FECD $(P=0.0462)$ (Figure 1F) (normal $n=3$, FECD $n=6$ ). Therefore, it is likely that activation of PINK1-phospho-Parkin leads to Drp1 degradation during mitophagy in FECD. Further, Parkin is known to undergo self-ubiquitination following phosphorylation. ${ }^{48}$ These findings are consistent with no significant differences in gene expression of Parkin and Drp1 between normal and FECD tissues (Supplemental Figure S1B).

\section{MN Induces Parkin Activation and Loss of Drp1 during Mitochondrial Fragmentation and Mitophagy}

Because FECD has been deemed an oxidative stress disorder, the mechanism of mitophagy activation via upregulation of intracellular ROS was further investigated. Intracellular ROS generation with MN leads to DNA damage and subsequent mitochondrial fragmentation and mitophagy. ${ }^{1,16}$ MN generates superoxide and unstable semiquinones due to one-electron reduction that induces morphological changes in cell monolayers of HCEnCs resulting in rosette formations, characteristic of FECD ex vivo. ${ }^{16}$ This feature mimics acellular center formation during CE cell apoptosis around guttae seen in FECD MN-induced decrease of $\Delta \psi \mathrm{m}$ and adenosine triphosphate levels has been reported in HCEnCs. ${ }^{16}$ The induction of intracellular ROS, specific to FECD, was modeled by treating normal HCEnCs with $\mathrm{MN}$ and observing 
A

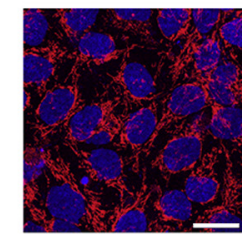

C
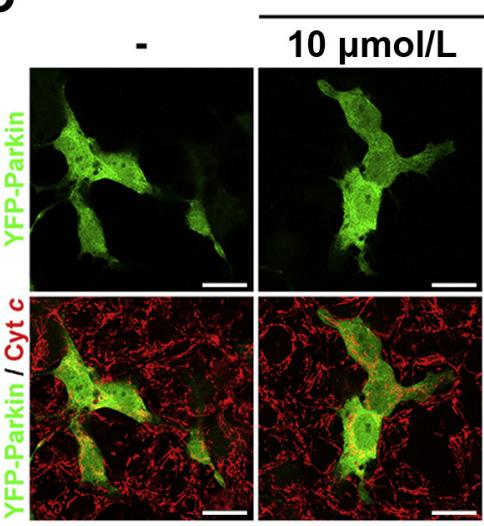

E

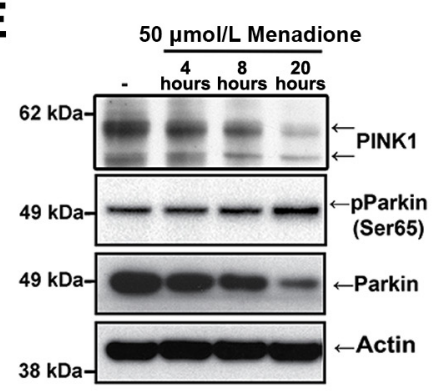

$\mathbf{F}$

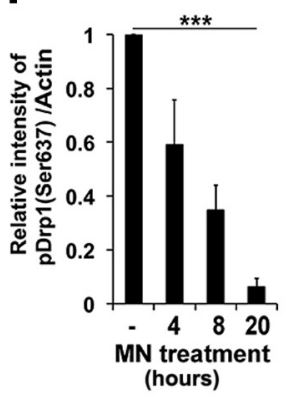

I

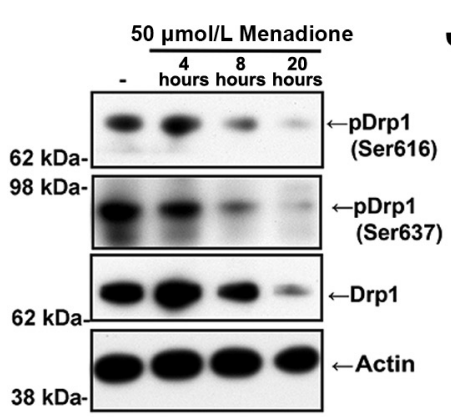

M

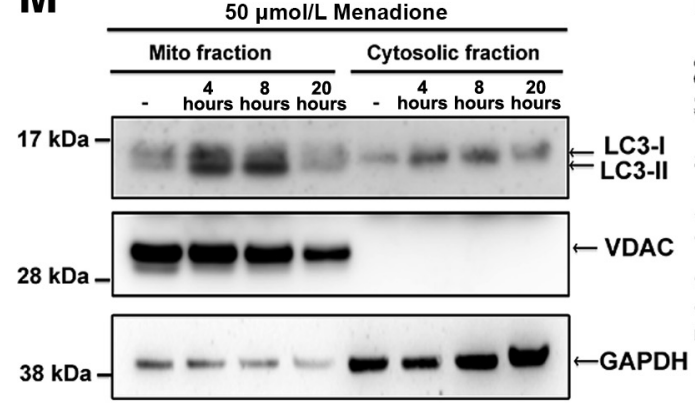

$\mathbf{N}$
Menadione $25 \mu \mathrm{mol} / \mathrm{L}$ $50 \mu \mathrm{mol} / \mathrm{L}$

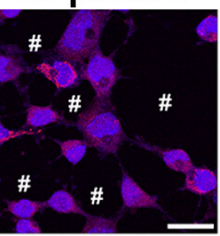

Menadione
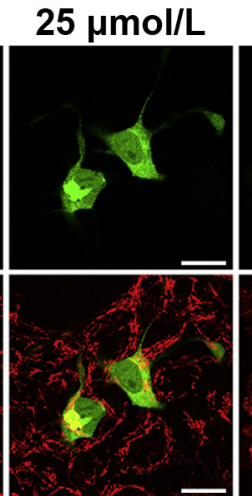

$50 \mu \mathrm{mol} / \mathrm{L}$

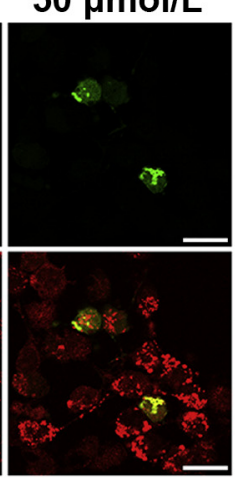

G

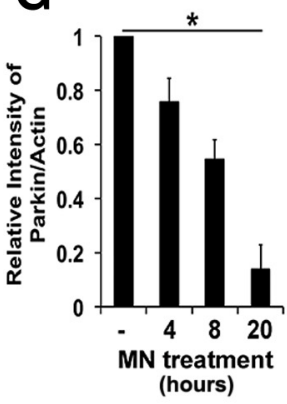

K
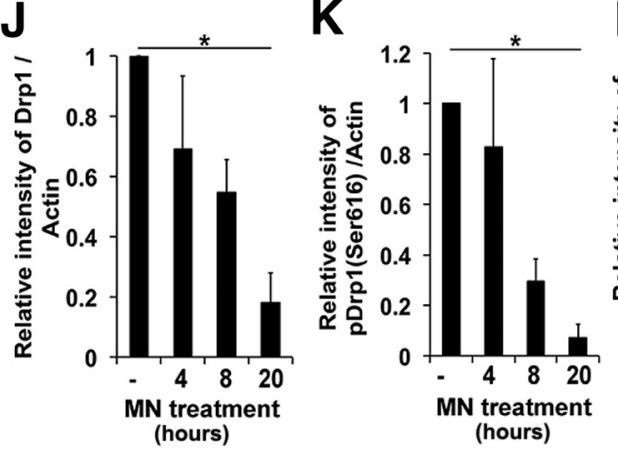

B
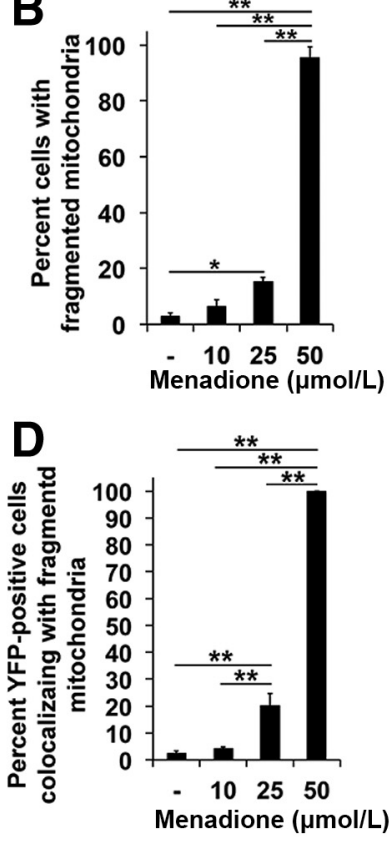

H

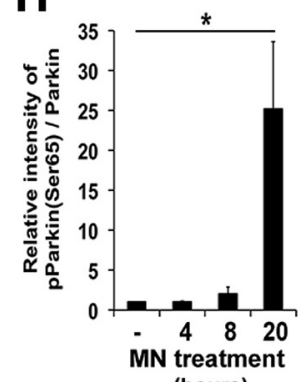

(hours)

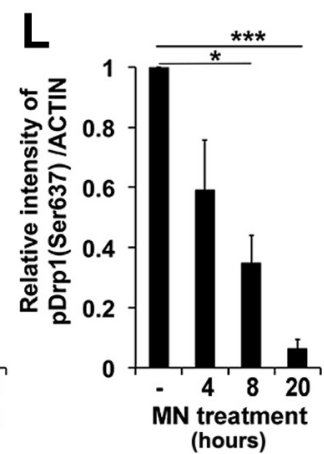

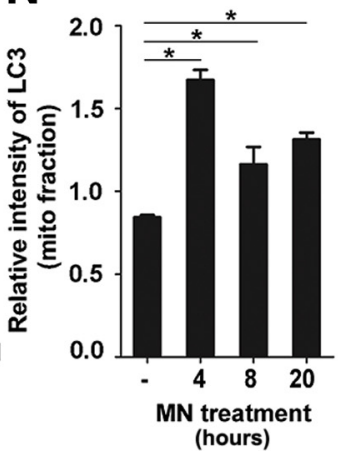

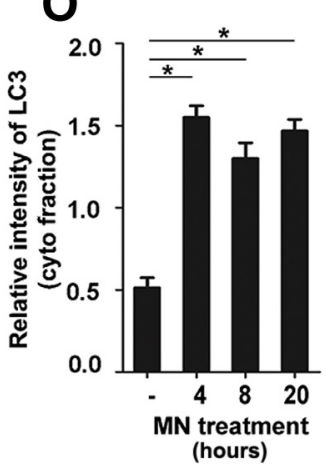


mitochondrial fragmentation during rosette formation by the immunolocalization of cyt $c$. MN-induced mitochondrial fragmentation starting at $25 \mu \mathrm{mol} / \mathrm{L}$ (approximately $17 \%$ cells) which further increased at $50 \mu \mathrm{mol} / \mathrm{L}$ ( $>90 \%$ cells) as detected by the loss of tubular mitochondrial networks and increased translocation of mitochondria to perinuclear regions (Figure 2, A and B). Treatment with escalating doses of $\mathrm{MN}$ resulted in the total abrogation of normal mitochondria around the rosettes (Figure 2A) with the release of cyt $c$ in approximately $95 \%$ of cells at $50 \mu \mathrm{mol} / \mathrm{L} \mathrm{MN}$ (Figure 2, A and B), indicating loss of mitochondrial mass as quantified in previous reports. ${ }^{49} \mathrm{~A}$ representative high magnification image of the loss of tubular mitochondrial network that was quantified in Figure $2 \mathrm{~B}$ is shown in Supplemental Figure S2A.

Because elevated Parkin phosphorylation was detected in FECD tissue, it was further investigated whether Parkin is involved in $\mathrm{MN}$-induced mitochondrial fragmentation. To visualize Parkin in relation to mitochondrial dynamics, exogenous YFP-Parkin was introduced to HCEnC-21T ${ }^{50}$ and treated cells with increasing doses of $\mathrm{MN}$ as reported. ${ }^{16}$ In the untreated cells, Parkin is mostly localized in the cytosol with only a small fraction detected in the mitochondria, as previously noted. ${ }^{51} \mathrm{MN}$ treatment resulted in a dose-dependent translocation of Parkin to fragmented mitochondria clustered in the perinuclear region, quantified as the percentage of YFP-Parkin-positive cells that colocalized with fragmented mitochondria. Significant increase in the colocalization of YFP-Parkin was observed with fragmented mitochondria starting at $25 \mu \mathrm{mol} / \mathrm{L} \mathrm{MN}$ (Figure 2D). The fragmented mitochondria appeared as perinuclear aggregates and showed maximal colocalization with YFP-Parkin at $50 \mu \mathrm{mol} / \mathrm{L} \mathrm{MN}$ (Figure 2, C and D). The enlarged image of Figure $2 \mathrm{C}$ is shown in Supplemental Figure S2B. It is reported that Parkin-mediated ubiquination triggers the perinuclear clustering of damaged mitochondria. ${ }^{52}$ These findings suggest that $\mathrm{MN}$ induces Parkin translocation during mitochondrial fragmentation, providing the dynamic model system of YFP Parkin colocalization with fragmented mitochondria in response to intracellular oxidative stress. Further, the endogenous levels of phosphoParkin were assessed in the mitochondrial fractions of HCEnC-21T cells treated with MN (Supplemental Figure S2C). Corroborating the immunostaining data, an increase in phospho-Parkin levels was detected in the mitochondrial fractions, indicating increased translocation of activated Parkin to mitochondria.

Next, the effect of MN on the endogenous mitophagyrelated proteins was investigated by Western blot analysis. HCEnC-21T cells treated with MN showed a timedependent decrease in PINK1 and total Parkin, and an increase in phospho-Parkin (Ser65) (Figure 2, E-H) in the whole-cell lysates. Reduction in total Parkin levels was detected as early as 4 hours after MN treatment, and levels progressively decreased with 8 and 20 hours of treatment (Figure 2G). Specifically, $82 \%$ and $86 \%$ decreases were detected in PINK1 $(P=0.02)$ and total Parkin $(P=0.02)$, respectively, at 20 hours of MN treatment compared with untreated cells (Figure 2, F and G). The occurrence of apoptosis at the later 20 hours time point as a result of the release of cyt $\mathrm{c}$ from the highly damaged mitochondria cannot be ruled out; however, the changes seen at the early time points of 4 hours and 8 hours clearly indicate mitophagy. Phospho-Parkin (Ser65) showed a 25-fold increase at 20 hours of $\mathrm{MN}$ when normalized to total endogenous Parkin (Figure 2, $\mathrm{E}$ and $\mathrm{H})(P=0.016)$. Similar to ex vivo findings, a temporal decrease was detected in total Drp1 $(82 \%, P=0.015)$. There was no change in the levels of phospho-Drp1 (Ser616) and phospho-Drp1 (Ser637) after 4 hours of MN treatment but eventually the phosphorylated forms, phospho-Drp1 (Ser616) (93\%, $P=0.028$ ) and phospho-Drp1 (Ser637) Drp1 (94\%, $P<0.001$ ), showed a decline from 8 hours of treatment as compared with no-treatment controls in the whole-cell lysates (Figure 2, I-L). These data hint at the possibility that ROS-mediated mitochondrial fragmentation does not necessarily activate Drp1. In fact, the protective function of Drp1 seen in normal mitochondrial homeostasis such as embryogenesis ${ }^{16,53,54}$ or mitosis, ${ }^{46}$ may be abrogated in the disease process herein. Therefore, a decline in Drp1 in FECD likely impedes fission-mediated restoration of lost $\Delta \psi \mathrm{m}$ in response to oxidative stress. Recently, a study showed that oxidative stress with $\mathrm{H}_{2} \mathrm{O}_{2}$ caused the hyperacetylation of microtubules (MT) and precluded activation of phospho-Drp1 (Ser616) during fission. ${ }^{55}$ Because $\mathrm{MN}$ induces protein acetylation ${ }^{56}$ the impact of such posttranslational modifications on fission/fusion balance in FECD needs further investigation.

Figure 2 Menadione-induced fragmentation of mitochondria and activation of mitophagy in HCECn-21T cells mimic observations from human specimens. A and B: Immunofluorescence localization of cyt $c$ (red) in HCEnC-21T cells treated with 10, 25, and $50 \mu \mathrm{mol} / \mathrm{L}$ menadione (MN) for 20 hours. Dashes indicate untreated control sample. The formation of rosettes in the far right panel $(\mathbf{A})$ is indicated by hash marks. The percentage of cells with fragmented mitochondria upon MN treatment is quantified in B. C: HCEnC-21T cells transfected with YFP-Parkin and treated with 10, 25, and 50 $\mu$ mol/L menadione for 20 hours. The number of cells transfected with YFP-Parkin (green) that colocalize with fragmented mitochondria are determined (cyt $c$ ). Dashes indicate untreated control sample. D: Approximately 300 YFP-positive cells were counted for each experimental condition in C. E-H: Western blot analysis of PINK1, Parkin, and phospho-Parkin (Ser65) in the whole-cell lysates of HCEnC-21T cells treated with $50 \mu \mathrm{mol} / \mathrm{L}$ menadione for various time points. I-L: Western blot of Drp1, phospho-Drp1 (Ser616), and phospho-Drp1 (Ser637) in the whole-cell lysates of HCEnC-21T cells treated with $50 \mu$ mol/L menadione. The decrease in phospho-Drp1 (Ser637) at 8 hours after MN is statistically significant. M-0: Western blot analysis of mitochondrial and cytosolic fractions of $50 \mu$ mol/L MN-treated HCEnC-21T cells for LC3-I/II for various time points. VDAC served as the mitochondrial fraction control, and GAPDH was used as cytosolic fraction control. The relative intensities of LC3 levels in the mitochondrial $(\mathbf{N})$ and cytosolic $(\mathbf{0})$ fractions are represented as bar graphs. Data are expressed as means \pm SEM. $n=3$ independent experiments (D-H, N, and $\mathbf{0}) .{ }^{*} P<0.05,{ }^{*} * P<0.01$, and ${ }^{*} * *<0.001$ (one-way analysis of variance with post-hoc Tukey HSD). Scale bars $=10 \mu \mathrm{m}$. 


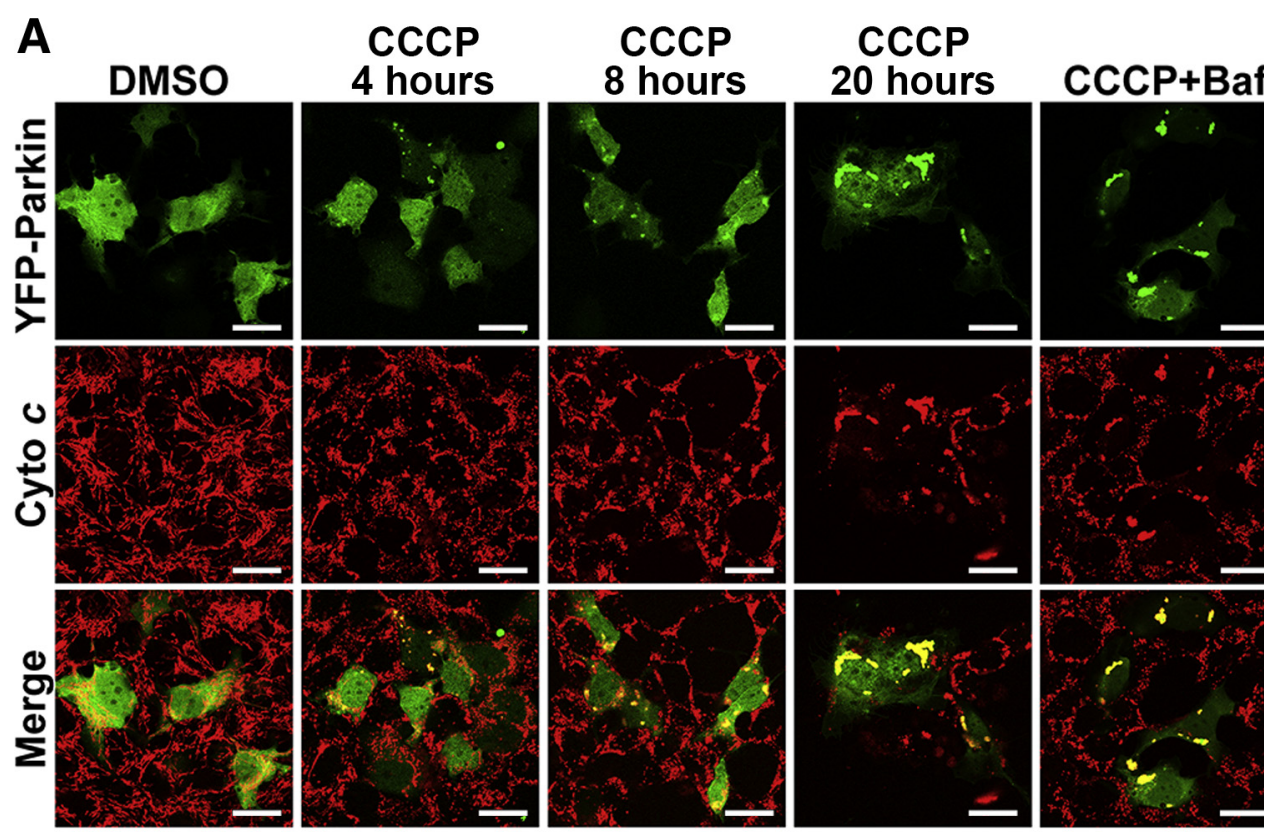

\section{B}

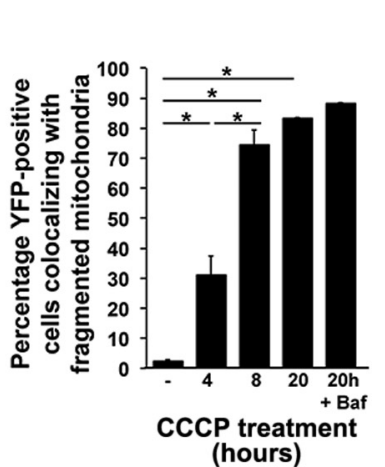

(hours)

C

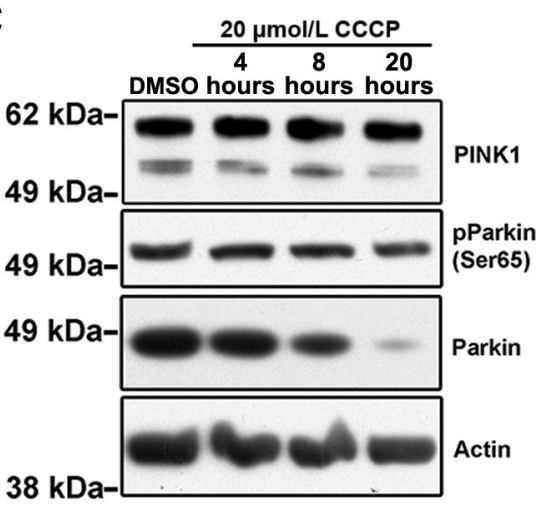

D E

E

$\mathbf{F}$
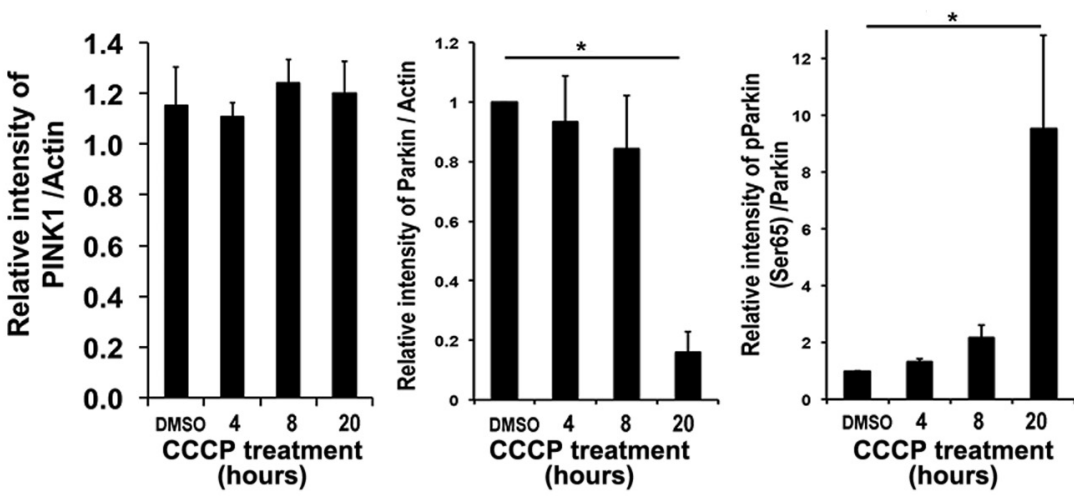

G

H

I

(hours)
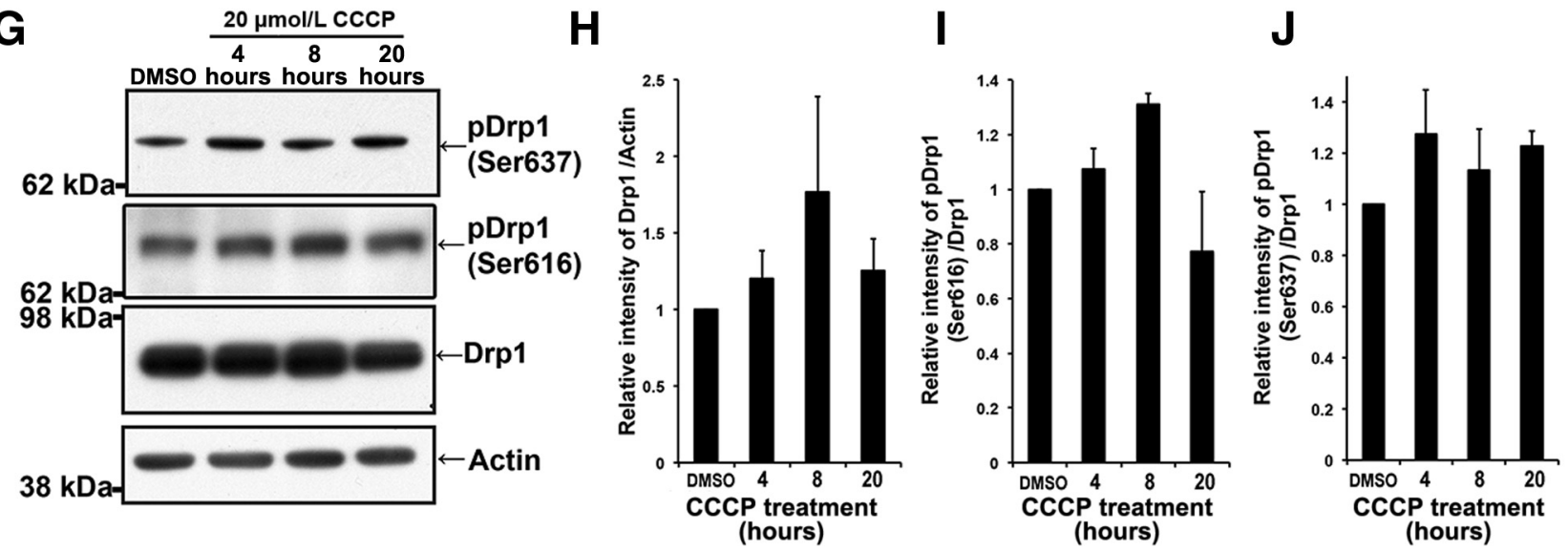

Figure 3 Mitochondrial fragmentation induced by CCCP reveals PINK1-independent phosphorylation of Parkin. A: Analysis of time-dependent translocation of YFP-Parkin (green) to fragmented mitochondria (red) in HCEnC-21T cells transfected with YFP-Parkin and treated with CCCP (20 $\mu \mathrm{mol} / \mathrm{L})$. Effect of bafilomycin (Baf) on the colocalization of YFP-Parkin-positive cells with fragmented mitochondria (cyt $c$ ). B: Quantification of the number of cells that exhibit colocalization of YFP with cyt c shown in A. C: Western blot analysis of Parkin and phospho-Parkin (Ser65) in the whole-cell lysates of HCEnC-21T cells treated with CCCP $(20 \mu \mathrm{mol} / \mathrm{L})$ in a time-dependent manner. D-F: Quantification of Western blots for PINK1, Parkin, and phospho-Parkin shown in C. G: Western blot of whole-cell lysates of HCEnC21T cells treated with CCCP $(20 \mu \mathrm{mol} / \mathrm{L})$ for Drp1, phospho-Drp1 (Ser616), and phospho-Drp1 (Ser637). H-J: Quantification of Western blots shown in G. Data are expressed as means \pm SEM. $n=3$ independent experiments $(\mathbf{D}-\mathbf{F})$. ${ }^{*} P<0.05$ (one-way analysis of variance with post-hoc Tukey HSD). Scale bar $=10 \mu \mathrm{m}$. 
Next, it was investigated whether oxidative stress triggers the downstream recruitment of autophagosomes to damaged mitochondria. The levels of autophagy marker LC3 were assessed specifically in the mitochondrial fractions isolated from MN-treated HCEnC-21T cells by Western blot analysis. Similar increases in the levels of LC3-II, the conjugated form of LC3-I recruited to the autophagosomal membrane, was noted starting at 4 hours and later at 8 and 20 hours after MN normalized to VDAC (Figure 2, M-O). The up-regulation of LC3 was also seen in the cytosolic fractions (Figure 2O). The purity of the mitochondrial and cytosolic fractions were verified using VDAC and glyceraldehyde-3-phosphate dehydrogenase (GAPDH) as markers, respectively. These data provide evidence for MNmediated ROS generation-induced autophagosome formation on the mitochondria of CE cells.

\section{CCCP Induces Phosphorylation of Endogenous Parkin during Mitochondrial Fragmentation in the $\mathrm{CE}$}

Because PINK1/Parkin is known to mainly respond to mitochondrial depolarization, the mechanisms of mitophagy activation were studied by uncoupling the $\Delta \psi \mathrm{m}$ via CCCP in $\mathrm{CE} .{ }^{28,57,58} \mathrm{CCCP}$ induced a time-dependent increase in the number of $\mathrm{CE}$ cells exhibiting fragmented mitochondria as shown by cyt $c$ localization in the perinuclear aggregates (Figure 3A). The enlarged image of Figure 3A is shown in Supplemental Figure S3. HCEnC-21T cells transfected with YFP-Parkin showed minimal colocalization of YFP with cyt $c$, whereas CCCP induced a time-dependent increase in the number of YFP-positive cells that showed a significant translocation to the mitochondrial aggregates (Figure 3, A and B). A significant increase in the colocalization of YFPParkin with fragmented mitochondria was noted as early as 4 hours of $20 \mu \mathrm{mol} / \mathrm{L} \mathrm{CCCP}$ treatment, which further increased at 8 hours and 20 hours of treatment.

A decline in Mfn2, the key fusion protein, is driven by auto/mitophagy and not proteasomal degradation in FECD. ${ }^{1}$ Immunofluorescence experiments in this study clearly demonstrated colocalization of Mfn2 and LC3 in the mitochondria with CCCP treatment providing direct evidence for active mitophagy. To further investigate the role of mitophagy-driven regulation of mitochondrial quality control proteins, bafilomycin $\mathrm{A} 1$, a vacuolar $\mathrm{H}^{+}$ATPase inhibitor that impedes the fusion between autophagosome and lysosome, was used to test its ability to inhibit mitochondrial fragmentation and translocation of Parkin to mitochondria. ${ }^{59}$ B A1 alone did not significantly change the number of YFPParkin-transfected cells that colocalized with fragmented mitochondria (1.8\% in dimethyl sulfoxide vs $4.2 \%$ in bafilomycin) $(P=0.17)$ (Supplemental Figure S4). The combination of CCCP and bafilomycin also did not rescue the fragmentation and translocation of YFP-Parkin due to CCCP, resulting in the increased percentage of YFP-Parkin cells colocalized to fragmented mitochondria compared with CCCP treatment only $(79.6 \%$ in CCCP vs $88 \%$ in
CCCP+bafilomycin, $P=0.0007$ ) (Figure $3, \mathrm{~A}$ and $\mathrm{B}$ ). These data suggest that blocking mitophagy did not affect the mitochondrial translocation of Parkin and mitochondrial fragmentation due to depolarizing stress.

Western blot analysis of HCEC-21T lysates treated with CCCP at different time points showed $84 \%$ decrease $(P=0.005)$ in endogenous Parkin and a reciprocal 9.5-fold increase $(P=0.027$ ) (Figure $3 \mathrm{~F})$ in phospho-Parkin (Ser65) compared with untreated cells (Figure 3, C, E, and F). By contrast, PINK1 remained unaffected in these conditions, suggesting that mitochondrial fragmentation stimulated by uncoupling of $\Delta \psi \mathrm{m}$ does not possibly involve PINK1 (Figure 3, C and D) in CE. Similar to PINK1, the protein levels of Drp1 and its two phosphorylated forms did not reveal any significant changes with increase in time of CCCP treatment (Figure 3, G-J). Although the total protein levels of PINK1 remained unchanged, it is possible that the activity of PINK1 increased in response to depolarization, warranting further activity studies in the future. Similarly for Drp1, it is possible that CCCP induced changes in the other post-translational modifications of Drp1 (S-nitrosylation, sumoylation), which were not tested in this study.

The translocation of Parkin to depolarized mitochondria has been shown to occur as early as 1 hour following treatment with CCCP and at 12 hours with valinomycin. ${ }^{51,60,61}$ The number of cells that show an aggregation of mitochondria with CCCP that colocalize with YFP-Parkin were quantified with a method that has been used previously. ${ }^{62}$ Approximately $30 \%$ of YFP-Parkin-transfected cells showed complete overlap with fragmented mitochondria at 4 hours with maximum percentage at 8 hours. This observation is concordant with the previous report where approximately $35 \%$ of transfected cells showed a complete overlap at 2 hours following stimulation with CCCP in HeLa cells transfected with GFP-Parkin. ${ }^{62}$ Furthermore, an increase was observed in endogenous phospho-Parkin (Ser65) that suggests a plausible PINK1-independent activation of Parkin. The differences in PINK1, Parkin, and Drp1 due to depolarizing (CCCP) and nondepolarizing stressors suggest the presence of alternative mechanisms for ROS-dependent and depolarizationdependent signaling. ${ }^{63}$ These results show two modalities of activation of mitophagy: one is stimulated by abundance of ROS, and the other results from uncoupling of proton gradient. Although it could be assumed that both methods of activation of mitophagy would follow a similar pathway, these results indicate that PINK1 and Drp1 levels remain unaffected with CCCP, whereas a loss of these proteins is observed with MN.

Parkin-Dependent Proteasomal Degradation Promotes Mitophagy and Protein Degradation in FECD

To further investigate the mechanism of Parkin involvement in FECD, immortalized CE cell lines derived from normal and FECD patient specimens, HCECi and FECDi, respectively, were chosen. ${ }^{43,44}$ A series of experiments were 

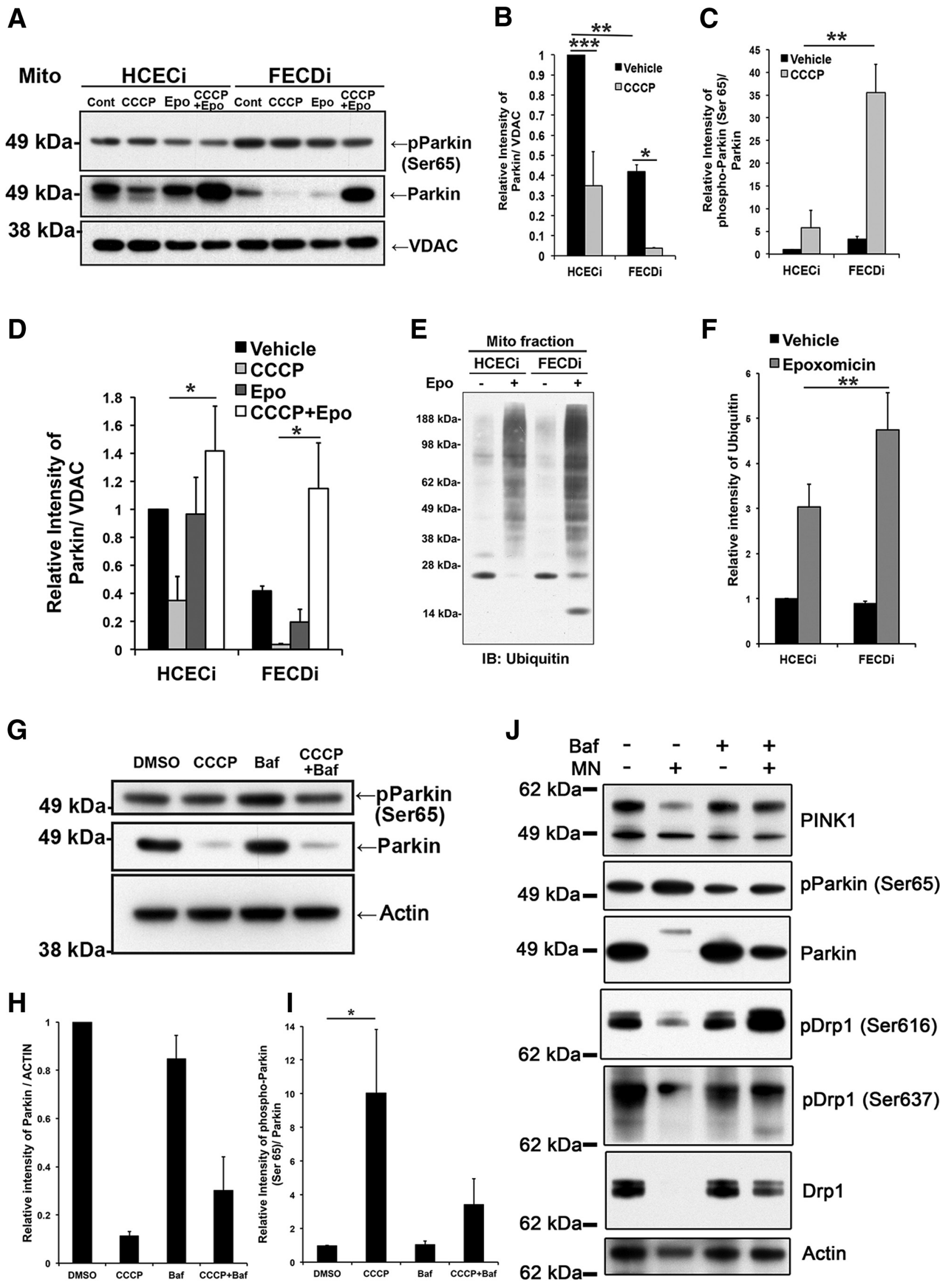
performed selectively inhibiting proteasomal degradation or mitophagy in response to loss of $\Delta \psi \mathrm{m}$ and oxidative stress with $\mathrm{MN}$ in these cell lines. Because heightened susceptibility of FECD to depolarizing stimuli is reported, ${ }^{1}$ normal (HCECi) and FECD (FECDi) cells were treated with CCCP and epoxomicin, a proteasomal inhibitor with high specificity, and the levels of phosphorylated and total Parkin were assessed in mitochondrial fractions. Intact mitochondria were isolated from these treated cell lines using antiTOM22 antibody-bound magnetic beads as previously reported. ${ }^{1}$ Lower total mitochondrial Parkin was detected in FECDi compared with HCECi (0.4-fold, $P=0.0016$ ) (Figure 4, A and B) and in $\mathrm{HCECi}$ treated with $\mathrm{CCCP}$ compared with untreated HCECi (0.3-fold, $P=0.0009$ ) (Figure 4, A and B). Treatment with CCCP almost completely obliterated Parkin levels in FECDi compared with its baseline (91\% decrease, $P=0.015$ ) (Figure 4, A and $\mathrm{B}$ ), consistent with ex vivo findings of total Parkin loss in FECD. Furthermore, there was a 6.2-fold greater upregulation of phospho-Parkin (Ser65) in FECDi compared with HCECi $(P=0.001)$ with CCCP, suggesting that FECDi exhibited an increased susceptibility to mitochondrial depolarization (Figure 4C). In HCECi, epoxomicin rescued CCCP-induced degradation of Parkin (CCCP 0.3fold vs CCCP+epoxomicin 1.4-fold; $P=0.027$ ) (Figure 4D). Similarly, a rescue of total Parkin was observed due to epoxomicin in FECDi (CCCP 0.04-fold vs $\mathrm{CCCP}+$ epoxomicin 1.1-fold; $P=0.021$ ) (Figure 4D). Comparison of the fold rescue of total Parkin due to epoxomicin and CCCP in HCECi (4-fold) and FECDi (30-fold) suggests a possible aberrant stimulation of the proteasomemediated degradation in FECDi (Figure 4D). To further ascertain this observation, the protein lysates of mitochondrial fractions from HCECi and FECDi probed for ubiquitin (Figure 4E) did not reveal significant difference between cell lines at baseline, but addition of epoxomicin led to a significantly greater ubiquitin staining intensity in FECDi (Figure 4, E and F) $(P=0.021)$, suggesting a greater rescue of mitochondrial proteins destined for degradation, hence, greater constitutive proteasomal degradation of overall proteins in FECD.

Because the inhibition of proteasome with epoxomicin rescued the degradation of Parkin, the effect of inhibition of autophagosome formation that blocks mitophagy, was studied on Parkin levels. Western blot analysis of CCCP- and bafilomycin-treated lysates of HCEnC-21T cells did not reveal a change in the levels of endogenous phospho-Parkin (Ser65) and total Parkin (Figure 4, G-I) compared with CCCP-treated HCEnC-21T cell lysates, consistent with the observation on mitochondrial fragmentation and translocation of YFP-Parkin due to bafilomycin (Figure 3, A and B). This observation also reaffirms that Parkin protein undergoes proteasome-mediated degradation (Figure 4A) upon CCCP treatment, and not mitophagy-dependent clearance. By contrast, bafilomycin rescued $\mathrm{MN}$-induced decrease in endogenous PINK1, total Parkin, total Drp1, and both isoforms of active Drp1, such as phospho-Drp1 (Ser616) and phospho-Drp1 (Ser637) (Figure 4J). Bafilomycin also reversed the increase in phospho-Parkin (Ser65) stimulated by $\mathrm{MN}$ (Figure $4 \mathrm{~J}$ ), indicating the overturning of Parkin activation. These results indicate a mitophagy-dependent degradation of endogenous proteins in response to oxidative stress. Specifically, we find that degradation of Parkin and constitutive loss of PINK1 and Drp1 are mitophagy-mediated when oxidation is the primary trigger in the disease process seen in FECD. These observations are consistent with the earlier reports showing that Parkin exhibits autoubiquitination activity following phosphorylation. ${ }^{27,29}$

\section{Discussion}

Previous study has shown that constitutive activation of mitophagy is a hallmark of degenerating CE cells in FECD. ${ }^{1}$ In this study, the authors demonstrate significant up-regulation of PINK1 and phospho-Parkin protein levels in the post-surgical FECD patient specimens compared with normal corneas. This study provides the first line of evidence on the mechanism of excessive mitochondrial clearance, where Parkin/PINK1-dependent mitophagy is activated by genetic and environmental factors involved in the pathogenesis of FECD (Figure 5). During rosette formation, mitochondrial fragmentation is a prominent feature of abating mitochondrial network, where PINK1 and phospho-Parkin recruitment leads to the activation of autophagosome formation (Figure 5). Although mitochondrial fragmentation was prominent in FECD, down-regulation of the key fission protein Drp1 was detected in FECD tissue samples and in the

\footnotetext{
Figure 4 FECDi endothelial cell line shows increased activation of Parkin that promotes mitochondrial protein degradation through ubiquitination. A-C: Representative Western blot of mitochondrial (Mito) fractions purified with anti-TOM22-coupled magnetic beads from HCECi and FECDi treated with dimethyl sulfoxide (DMSO), CCCP $(20 \mu \mathrm{mol} / \mathrm{L})$, epoxomicin $(1 \mu \mathrm{mol} / \mathrm{L})$, or both CCCP and epoxomicin (Epo) for 6 hours probed for phospho-Parkin (Ser65) and Parkin. B and C: Quantification of the relative intensities of Parkin and phospho-Parkin (Ser65) from the Western blots shown in A normalized with the mitochondrial marker VDAC. D: Densitometric quantification of total Parkin by Western blot analysis to study the effect of epoxomicin on the CCCP-induced degradation of Parkin in HCECi and FECDi cells. E: Representative Western blot of the mitochondrial fractions purified with anti-TOM22-coupled magnetic beads of vehicleand epoxomicin-treated HCECi and FECDi cells probed for ubiquitin. F: Quantification of the Western blot shown in E normalized with VDAC. G-I: Western blot analysis of Parkin, phospho-Parkin (Ser65) in HCEnC-21T whole-cell lysates treated with CCCP (20 $\mu \mathrm{mol} / \mathrm{L})$, bafilomycin (Baf) (10 nmol/L), and CCCP + Baf for 20 hours. J: Analysis of the effect of bafilomycin in MN-treated HCEnC-21T cells on the protein levels of PINK1, Parkin, Drp1, phospho-Drp1 (Ser616), and phospho-Drp1 (Ser637) determined by Western blot analysis of the whole-cell lysates. Data are expressed as means \pm SEM (D, one-way analysis of variance with post-hoc Tukey HSD; B, C, and $\mathbf{F}$, two-way analysis of variance with post-hoc Tukey HSD). $n=3$ independent experiments $(\mathbf{B}-\mathbf{D}, \mathbf{F}, \mathbf{H}$, and $\mathbf{I}) .{ }^{*} P<0.05$, ${ }^{* *} P<0.01$, and ${ }^{* * *} P<0.001$. Cont, untreated control sample; MN, menadione.
} 


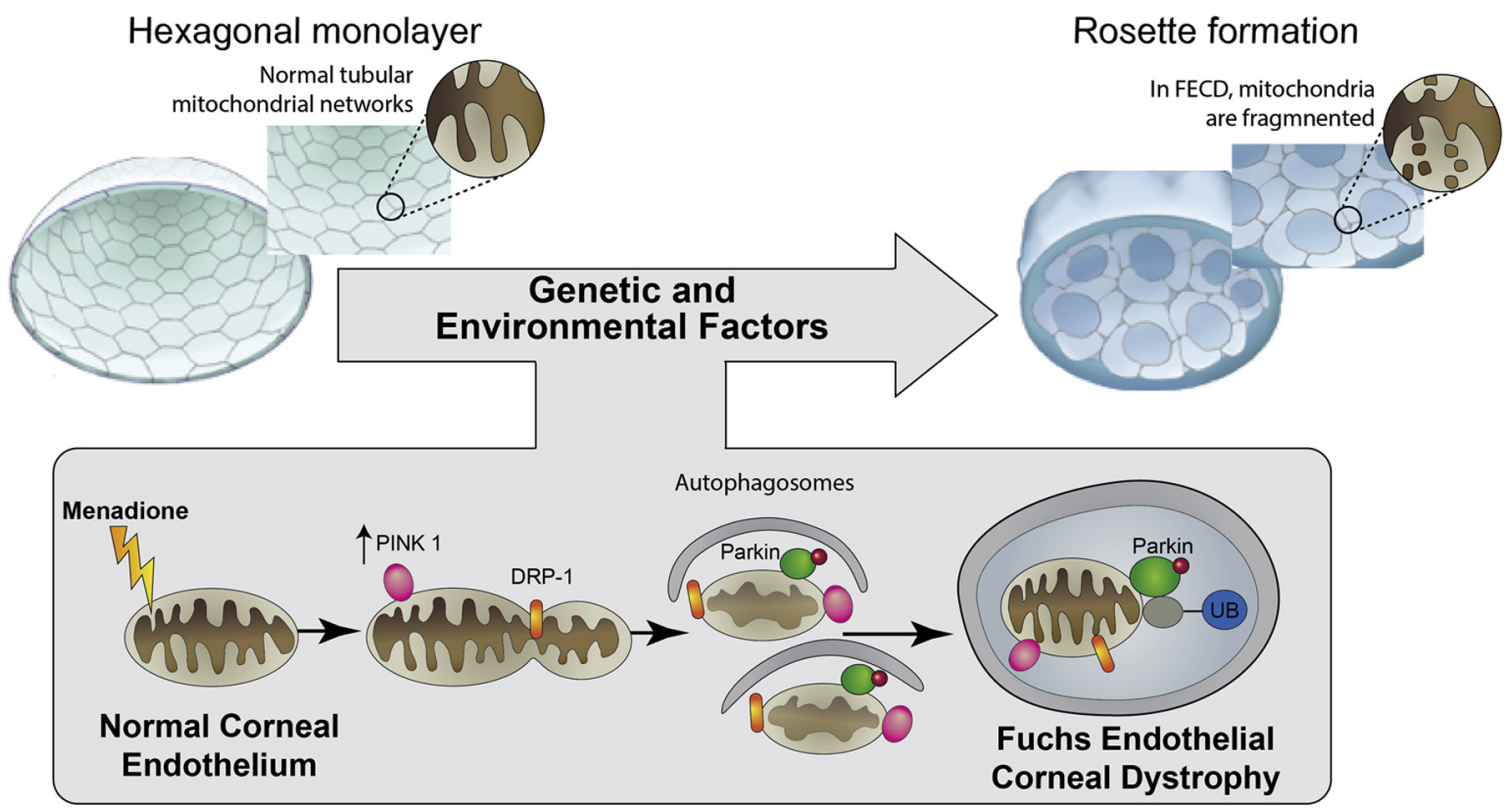

Figure 5 Mitophagy drives endogenous protein clearance in FECD. Schematic of the mechanism of mitochondrial fragmentation and mitophagy in the pathogenesis of Fuchs endothelial corneal dystrophy (FECD). Menadione induces endogenous oxidative stress and leads to Parkin-dependent mitophagy, sequestering Parkin, Drp1, and PINK1 during rosette formation seen in FECD.

in vitro model of ROS-induced mitophagy. To the best of our knowledge, a concurrent increase in the phospho-Parkin (Ser65) and decline in Drp1 during fission-induced mitophagy has not been reported; thus, providing an insight into the mechanism of mitophagy in the diseased post-mitotic cells. The limitation of using surgically removed specimens is that they provide information on the late stage of the disease; however, the advent of less invasive corneal transplantation methods ${ }^{64}$ has enabled access to human tissues at earlier stages, creating an opportunity to understand the innate pathogenic changes pertaining to the disease and subsequently modeling them in the experimental systems. Similar to our findings, recent reports in yeast and mammals show that Drp1 is not required for mitophagy, ${ }^{65-67}$ and mitochondrial fragmentation concurrently occurs with autophagosome formation leading to mitophagy in a Drp1-independent manner. ${ }^{68}$

The modeling of rosette formation with intracellular oxidative stress in vitro showed that MN induced Parkindependent mitophagy, where mitochondrial quality control proteins were recruited onto mitochondria and degraded by mitophagy and ubiquitination (Figure 5). The significance of this study is twofold. First, this study reports that MN stimulates the phosphorylation of endogenous Parkin and leads to up-regulation of Parkin-mediated mitophagy in post-mitotic cells of the eye. Most of the studies on the mechanism of PINK1- and Parkin-mediated mitophagy have utilized the overexpression of recombinant Parkin in various mitotically competent cells. ${ }^{26-28}$ Although, studies have shown an increase in phospho-Parkin (Ser65) due to loss of $\Delta \psi \mathrm{m},{ }^{28,29}$ the phosphorylation of endogenous Parkin due to MN-induced ROS has not been reported. A recent study showed that hydrogen peroxide treatment alone failed to induce translocation of Parkin to mitochondria and activate mitophagy, unless mitochondrial depolarization with CCCP jumpstarted the process in HeLa cells. ${ }^{49}$ By contrast, a robust ROS-dependent translocation of YFP-Parkin to fragmented mitochondria and up-regulation of endogenous phospho-Parkin (Ser65) in response to MN was observed. Further, the activation of Parkin triggers the autophagosome formation around mitochondria evidenced by LC3-II accumulation in the mitochondrial fractions. These data indicate that ROS can serve as a primary trigger of Parkin-induced mitophagy and can act upstream of Parkin. MN induces mitochondrial superoxide that eventually leads to uncoupling of $\Delta \psi \mathrm{m}$ during rosette formation, ${ }^{16}$ demonstrating that MN likely exerts the combination of signaling effects of ROS and mitochondrial depolarization, and simulates the physiological stressors encountered in the disease process. Therefore, our results connect the downstream effect of mitochondrial damage with ROS-induced activation of Parkin and mitophagy seen in FECD. ${ }^{1}$

Second, oxidative stress led to loss of endogenous PINK1 production, despite Parkin-activated mitochondrial fragmentation. Previous studies performed with underexpression of PINK1, ${ }^{69-71}$ overexpression of mutant forms ${ }^{72}$ or with cell lines containing endogenously mutated PINK $1{ }^{61}$ have shown PINK1 to be protective during cellular stress. Engineered loss of PINK1 in neuronal cells led to mitochondrial fragmentation and mitophagy depending on mitochondrial oxidant production. ${ }^{69}$ Unique to our study, down-regulation of endogenous PINK1 (in nonmutated human cell lines) was achieved by generation of mitochondrial superoxide during rosette formation, ${ }^{16}$ indicating 
that ROS may be involved in PINK1-independent activation of mitophagy. The difference between ex vivo and in vitro findings could be due to the fact that activation of PINK1 in tissue represents the end stage of the disease where mitochondrial depolarization is a predominant trigger of mitophagy ${ }^{1}$ as opposed to ROS being a primary trigger of $\mathrm{MN}$-induced mitophagy in vitro. Moreover, cellular-matrix interactions preserved in the specimens ${ }^{13,39,40}$ and the decades of chronic stress throughout the disease process therein could be other contributing factors to the differential findings. A recent study reports the recruitment of Parkin to mitochondria and induction of mitophagy in the cardiac myocytes of PINK1-deficient mice $\left(\right.$ Pink1 $\left.^{-/-}\right)$upon treatment with mitochondrial uncoupler FCCP. ${ }^{73}$ Similarly, in Drosophila melanogaster, Parkin rescues the mitochondrial defects shown by PINK1 mutants, indicating that PINK1 is dispensable for Parkin activation. ${ }^{74}$

Despite the mitochondrial fragmentation in FECD, significant down-regulation of total Drp1 levels were detected that could be attributed to the proteasomal degradation by activated Parkin. Similar to our findings, Alzheimer brain specimens showed reduction in mitochondrial fusion/fission proteins, including Drp1, consistent with altered mitochondrial dynamics in hippocampal neurons during neurodegeneration. ${ }^{75}$ However, because phosphorylation is one of the many post-translational modifications of Drp1, the possibility that other modifications of Drp1 such as S-nitrosylation, sumoylation, or ubiquitination are involved in aberrant mitochondrial dynamics in FECD cannot be ruled out. Similarly, drastic reduction was observed in total Parkin protein levels, despite an increase in activated phosphoParkin. Parkin is a RING-type E3 ligase, and its N-terminal UBL domain inhibits its autoubiquitination under normal cellular conditions that would otherwise lead to its proteasomal degradation. Upon mitochondrial depolarization, PINK1-mediated phosphorylation of Ser65 in the UBL domain opens up the autoinhibited conformation of Parkin, leading to its activation and subsequent self-ubiquitination. ${ }^{48,76,77}$ Upon mitochondrial depolarization, activated Parkin causes the turnover of outer mitochondrial proteins via ubiquitination, including its own degradation. This is reflected in the increased levels of ubiquitinated proteins noted in the mitochondrial fractions of FECDi upon treatment with proteasomal inhibitor, epoxomicin (Figure 4E) owing to increased phospho-Parkin. This is further supported by the recovery of total Parkin levels upon the addition of epoxomicin with CCCP treatment. The extent of recovery of Parkin was higher in FECDi cells upon epoxomicin + CCCP treatment, indicating increased stimulation of proteasomal degradation in FECD. Therefore, decreased protein levels of Drp1 are likely due to Parkin-mediated ubiquitination and proteasomal degradation of mitochondrial proteins. ${ }^{47}$

Parkin is known to regulate the degradation of mitochondrial proteins by two distinct pathways: proteasomal degradation and mitophagy-dependent clearance. ${ }^{34}$ Interestingly, ROS-induced changes in the mitochondrial quality control proteins upon MN treatment could be rescued by blocking mitophagy using bafilomycin. By contrast, bafilomycin could not rescue the changes in Parkin levels upon mitochondrial depolarization induced by CCCP. Instead, the reduction in total Parkin due to $\mathrm{CCCP}$ was rescued by blocking the proteasomal degradation pathway using epoxomicin. These data suggest differences in the downstream pathways of Parkin-mediated mitochondrial clearance in response to ROS versus mitochondrial depolarization. Although ROS activated mitophagy-dependent clearance, mitochondrial depolarization with CCCP promoted proteasomal degradation of mitochondrial proteins. These observations are consistent with the findings that ROS promotes the completion of mitophagy following mitochondrial translocation of Parkin. ${ }^{49}$ However, it is possible that addition of bafilomycin, the inhibitor of vacuolar-type $\mathrm{H}^{+}$-ATPase, could further reduce already low mitochondrial ATP levels from CCCP treatment, thus depleting the levels required for protein phosphorylation and restoration of phosphorylated proteins.

These results shed light on different mechanism by which CCCP and MN exert their effects on mitophagy activation. The majority of studies have used CCCP to depolarize $\Delta \psi \mathrm{m}$ in transfected cell lines and have shown the accumulation of PINK1 and its downstream activation of Parkin by phosphorylation. ${ }^{28,31,78}$ As evidenced by this current study, the biological relevance of solely using mitochondrial uncouplers when studying endogenous disease processes may not fully represent the findings ex vivo, and pose several challenges. First, uncouplers cause depolarization of entire mitochondrial network ${ }^{23}$; however, some may be preserved depending on the nature and cellular distribution of the disease. For example, mitochondrial fragmentation was found to be more prominent in the cells surrounding the rosettes, suggesting a topographical variability in mitochondrial depolarization in the FECD tissue. ${ }^{16}$ Second, uncouplers alone may not fully reflect the complexity of signaling pathways stemming from upstream factors such as oxidant-antioxidant imbalance, DNA damage, and/or unfolded protein response. ${ }^{8,16,79-81}$ Because $\mathrm{MN}$ is normally neutralized by 2-electron reduction by NQO1 and NQO1 is largely deficient in FECD, the MN-induced oxidative stress is fundamentally relevant for the study of oxidant-antioxidant imbalance stemming from deficient Nrf2-NQO1 axis seen in FECD. Similarly, Wang et al ${ }^{82}$ proposed that introducing short bursts of mitochondrial superoxide with mitochondrial KillerRed is a biologically relevant model for study of mitophagy in the context of oxidative stress disorders such as Parkinson and Alzheimer diseases. Further studies are warranted to study the upstream mechanisms involved in mediating the phospho-Parkin activation upon ROS stress.

The findings that auto/mitophagy is excessively up-regulated in FECD have a physiological significance for development of therapeutic strategies. Previous studies have postulated that lithium is cytoprotective in FECD by 
inducing autophagy and aiding in clearance of misfolded proteins. ${ }^{83}$ Although auto/mitophagy may be beneficial in general cellular survival, further stimulation of already excessive mitochondrial clearance may be detrimental to the bioenergetics of the cell in FECD. The findings that ROS act as upstream regulators of Parkin activation may provide a novel route of regulating protein degradation and mitochondrial dynamics, thus, restoring the bioenergetics of indispensable post-mitotic cells. Further studies are needed to investigate which ROS mediators would be most beneficial in the treatment of FECD.

\section{Acknowledgments}

We thank Peter Mallen for help in preparing the schematic in Figure 5; and Dr. May Griffith (Ottawa Hospital Research Institute, Ottawa, ON, Canada) and Dr. Rajiv Mohan (University of Missouri health System, Columbia, MO) for kindly providing the $\mathrm{CE}$ cell lines derived from normal and FECD patients' CE, respectively.

T.M., S.V., G.M., N.D., V.K., A.S.B., Y.C., and U.V.J. designed and performed experiments, and analyzed data; M.O.P., F.W.P., and U.V.J. provided human specimens used in this study; T.M., S.V., G.M., and U.V.J. prepared the manuscript; all authors revised the manuscript.

\section{Supplemental Data}

Supplemental material for this article can be found at http://doi.org/10.1016/j.ajpath.2019.06.012.

\section{References}

1. Benischke AS, Vasanth S, Miyai T, Katikireddy KR, White T, Chen Y, Halilovic A, Price M, Price F Jr, Liton PB, Jurkunas UV: Activation of mitophagy leads to decline in Mfn2 and loss of mitochondrial mass in Fuchs endothelial corneal dystrophy. Sci Rep 2017, 7:6656

2. Waring GO 3rd, Bourne WM, Edelhauser HF, Kenyon KR: The corneal endothelium. Normal and pathologic structure and function. Ophthalmology 1982, 89:531-590

3. Wieben ED, Aleff RA, Tosakulwong N, Butz ML, Highsmith WE, Edwards AO, Baratz KH: A common trinucleotide repeat expansion within the transcription factor 4 (TCF4, E2-2) gene predicts Fuchs corneal dystrophy. PLoS One 2012, 7:e49083

4. Dorrepaal SJ, Cao KY, Slomovic AR: Indications for penetrating keratoplasty in a tertiary referral centre in Canada, 1996-2004. Can J Ophthalmol 2007, 42:244-250

5. Ghosheh FR, Cremona FA, Rapuano CJ, Cohen EJ, Ayres BD Hammersmith KM, Raber IM, Laibson PR: Trends in penetrating keratoplasty in the United States 1980-2005. Int Ophthalmol 2008, 28:147-153

6. Gain P, Jullienne R, He Z, Aldossary M, Acquart S, Cognasse F, Thuret G: Global survey of corneal transplantation and eye banking. JAMA Ophthalmol 2016, 134:167-173

7. Azizi B, Ziaei A, Fuchsluger T, Schmedt T, Chen Y, Jurkunas UV: p53-regulated increase in oxidative-stress-induced apoptosis in Fuchs endothelial corneal dystrophy: a native tissue model. Invest Ophthalmol Vis Sci 2011, 52:9291-9297
8. Jurkunas UV, Bitar MS, Funaki T, Azizi B: Evidence of oxidative stress in the pathogenesis of Fuchs endothelial corneal dystrophy. Am J Pathol 2010, 177:2278-2289

9. Liu C, Chen Y, Kochevar IE, Jurkunas UV: Decreased DJ-1 leads to impaired Nrf2-regulated antioxidant defense and increased UV-A-induced apoptosis in corneal endothelial cells. Invest Ophthalmol Vis Sci 2014, 55:5551-5560

10. Ziaei A, Schmedt T, Chen Y, Jurkunas UV: Sulforaphane decreases endothelial cell apoptosis in Fuchs endothelial corneal dystrophy: a novel treatment. Invest Ophthalmol Vis Sci 2013, 54:6724-6734

11. Wojcik KA, Kaminska A, Blasiak J, Szaflik J, Szaflik JP: Oxidative stress in the pathogenesis of keratoconus and Fuchs endothelial corneal dystrophy. Int J Mol Sci 2013, 14:19294-19308

12. Bitar MS, Liu C, Ziaei A, Chen Y, Schmedt T, Jurkunas UV: Decline in DJ-1 and decreased nuclear translocation of Nrf2 in Fuchs endothelial corneal dystrophy. Invest Ophthalmol Vis Sci 2012, 53: 5806-5813

13. Jurkunas UV, Rawe I, Bitar MS, Zhu C, Harris DL, Colby K Joyce NC: Decreased expression of peroxiredoxins in Fuchs' endothelial dystrophy. Invest Ophthalmol Vis Sci 2008, 49: 2956-2963

14. Tuberville AW, Wood TO, McLaughlin BJ: Cytochrome oxidase activity of Fuchs' endothelial dystrophy. Curr Eye Res 1986, 5:939-947

15. Gottsch JD, Bowers AL, Margulies EH, Seitzman GD, Kim SW, Saha S, Jun AS, Stark WJ, Liu SH: Serial analysis of gene expression in the corneal endothelium of Fuchs' dystrophy. Invest Ophthalmol Vis Sci 2003, 44:594-599

16. Halilovic A, Schmedt T, Benischke AS, Hamill C, Chen Y, Santos JH, Jurkunas UV: Menadione-induced DNA damage leads to mitochondrial dysfunction and fragmentation during rosette formation in Fuchs endothelial corneal dystrophy. Antioxid Redox Signal 2016, 24:1072-1083

17. Youle RJ, van der Bliek AM: Mitochondrial fission, fusion, and stress. Science 2012, 337:1062-1065

18. Smirnova E, Griparic L, Shurland DL, van der Bliek AM: Dynaminrelated protein Drp1 is required for mitochondrial division in mammalian cells. Mol Biol Cell 2001, 12:2245-2256

19. Mizumura K, Cloonan SM, Nakahira K, Bhashyam AR, Cervo M, Kitada T, Glass K, Owen CA, Mahmood A, Washko GR, Hashimoto S, Ryter SW, Choi AM: Mitophagy-dependent necroptosis contributes to the pathogenesis of COPD. J Clin Invest 2014, 124:3987-4003

20. Kashatus DF, Lim KH, Brady DC, Pershing NL, Cox AD, Counter CM: RALA and RALBP1 regulate mitochondrial fission at mitosis. Nat Cell Biol 2011, 13:1108-1115

21. Chang CR, Blackstone C: Cyclic AMP-dependent protein kinase phosphorylation of Drp1 regulates its GTPase activity and mitochondrial morphology. J Biol Chem 2007, 282:21583-21587

22. Zhou L, Zhang Q, Zhang P, Sun L, Peng C, Yuan Z, Cheng J: c-Ablmediated Drp1 phosphorylation promotes oxidative stress-induced mitochondrial fragmentation and neuronal cell death. Cell Death Dis 2017, 8:e3117

23. Cereghetti GM, Stangherlin A, Martins de Brito O, Chang CR Blackstone C, Bernardi P, Scorrano L: Dephosphorylation by calcineurin regulates translocation of Drp1 to mitochondria. Proc Natl Acad Sci U S A 2008, 105:15803-15808

24. Santel A, Fuller MT: Control of mitochondrial morphology by a human mitofusin. J Cell Sci 2001, 114:867-874

25. Okatsu $\mathrm{K}$, Oka $\mathrm{T}$, Iguchi $\mathrm{M}$, Imamura $\mathrm{K}$, Kosako $\mathrm{H}$, Tani $\mathrm{N}$, Kimura M, Go E, Koyano F, Funayama M, Shiba-Fukushima K, Sato S, Shimizu H, Fukunaga Y, Taniguchi H, Komatsu M, Hattori N, Mihara K, Tanaka K, Matsuda N: PINK1 autophosphorylation upon membrane potential dissipation is essential for Parkin recruitment to damaged mitochondria. Nat Commun 2012, 3:1016

26. Narendra DP, Jin SM, Tanaka A, Suen DF, Gautier CA, Shen J, Cookson MR, Youle RJ: PINK1 is selectively stabilized on impaired mitochondria to activate Parkin. PLoS Biol 2010, 8:e1000298 
27. Kane LA, Lazarou M, Fogel AI, Li Y, Yamano K, Sarraf SA, Banerjee S, Youle RJ: PINK1 phosphorylates ubiquitin to activate Parkin E3 ubiquitin ligase activity. J Cell Biol 2014, 205:143-153

28. Kondapalli C, Kazlauskaite A, Zhang N, Woodroof HI, Campbell DG, Gourlay R, Burchell L, Walden H, Macartney TJ, Deak M, Knebel A, Alessi DR, Muqit MM: PINK1 is activated by mitochondrial membrane potential depolarization and stimulates Parkin E3 ligase activity by phosphorylating Serine 65. Open Biol 2012, 2:120080

29. Sha D, Chin LS, Li L: Phosphorylation of parkin by Parkinson disease-linked kinase PINK1 activates parkin E3 ligase function and NF-kappaB signaling. Hum Mol Genet 2010, 19:352-363

30. Koyano F, Okatsu K, Kosako H, Tamura Y, Go E, Kimura M, Kimura Y, Tsuchiya H, Yoshihara H, Hirokawa T, Endo T, Fon EA, Trempe JF, Saeki Y, Tanaka K, Matsuda N: Ubiquitin is phosphorylated by PINK1 to activate parkin. Nature 2014, 510:162-166

31. Kazlauskaite A, Kondapalli C, Gourlay R, Campbell DG, Ritorto MS, Hofmann K, Alessi DR, Knebel A, Trost M, Muqit MM: Parkin is activated by PINK1-dependent phosphorylation of ubiquitin at Ser65. Biochem J 2014, 460:127-139

32. Nixon RA: The role of autophagy in neurodegenerative disease. Nat Med 2013, 19:983-997

33. Chen Y, Dorn GW 2nd: PINK1-phosphorylated mitofusin 2 is a Parkin receptor for culling damaged mitochondria. Science 2013, 340:471-475

34. Yoshii SR, Kishi C, Ishihara N, Mizushima N: Parkin mediates proteasome-dependent protein degradation and rupture of the outer mitochondrial membrane. J Biol Chem 2011, 286:19630-19640

35. Gao J, Wang L, Liu J, Xie F, Su B, Wang X: Abnormalities of mitochondrial dynamics in neurodegenerative diseases. Antioxidants (Basel) 2017, 6:E25

36. Lazarou M, Sliter DA, Kane LA, Sarraf SA, Wang C, Burman JL, Sideris DP, Fogel AI, Youle RJ: The ubiquitin kinase PINK1 recruits autophagy receptors to induce mitophagy. Nature 2015, 524: 309-314

37. Suresh S, Raghu D, Karunagaran D: Menadione (Vitamin K3) induces apoptosis of human oral cancer cells and reduces their metastatic potential by modulating the expression of epithelial to mesenchymal transition markers and inhibiting migration. Asian Pac J Cancer Prev 2013, 14:5461-5465

38. Katikireddy KR, White TL, Miyajima T, Vasanth S, Raoof D, Chen Y, Price MO, Price FW, Jurkunas UV: NQO1 downregulation potentiates menadione-induced endothelial-mesenchymal transition during rosette formation in Fuchs endothelial corneal dystrophy. Free Radic Biol Med 2018, 116:19-30

39. Jurkunas UV, Bitar M, Rawe I: Colocalization of increased transforming growth factor-beta-induced protein (TGFBIp) and Clusterin in Fuchs endothelial corneal dystrophy. Invest Ophthalmol Vis Sci 2009, 50:1129-1136

40. Jurkunas UV, Bitar MS, Rawe I, Harris DL, Colby K, Joyce NC: Increased clusterin expression in Fuchs' endothelial dystrophy. Invest Ophthalmol Vis Sci 2008, 49:2946-2955

41. Price MO, Giebel AW, Fairchild KM, Price FW Jr: Descemet's membrane endothelial keratoplasty: prospective multicenter study of visual and refractive outcomes and endothelial survival. Ophthalmology 2009, 116:2361-2368

42. Schmedt T, Chen Y, Nguyen TT, Li S, Bonanno JA, Jurkunas UV: Telomerase immortalization of human corneal endothelial cells yields functional hexagonal monolayers. PLoS One 2012, 7:e51427

43. Griffith M, Osborne R, Munger R, Xiong X, Doillon CJ, Laycock NL, Hakim M, Song Y, Watsky MA: Functional human corneal equivalents constructed from cell lines. Science 1999, 286:2169-2172

44. He Y, Weng J, Li Q, Knauf HP, Wilson SE: Fuchs' corneal endothelial cells transduced with the human papilloma virus E6/E7 oncogenes. Exp Eye Res 1997, 65:135-142

45. Chang CR, Blackstone C: Drp1 phosphorylation and mitochondrial regulation. EMBO Rep 2007, 8:1088-1089. author reply 1089-1090
46. Taguchi N, Ishihara N, Jofuku A, Oka T, Mihara K: Mitotic phosphorylation of dynamin-related GTPase Drp1 participates in mitochondrial fission. J Biol Chem 2007, 282:11521-11529

47. Wang H, Song P, Du L, Tian W, Yue W, Liu M, Li D, Wang B, Zhu Y, Cao C, Zhou J, Chen Q: Parkin ubiquitinates Drp1 for proteasome-dependent degradation: implication of dysregulated mitochondrial dynamics in Parkinson disease. J Biol Chem 2011, 286:11649-11658

48. Zhang Y, Gao J, Chung KK, Huang H, Dawson VL, Dawson TM: Parkin functions as an E2-dependent ubiquitin- protein ligase and promotes the degradation of the synaptic vesicle-associated protein, CDCrel-1. Proc Natl Acad Sci U S A 2000, 97:13354-13359

49. Xiao B, Deng X, Lim GGY, Xie S, Zhou ZD, Lim KL, Tan EK: Superoxide drives progression of Parkin/PINK1-dependent mitophagy following translocation of Parkin to mitochondria. Cell Death Dis 2017, 8:e3097

50. Schmedt T, Silva MM, Ziaei A, Jurkunas U: Molecular bases of corneal endothelial dystrophies. Exp Eye Res 2012, 95:24-34

51. Narendra D, Tanaka A, Suen DF, Youle RJ: Parkin is recruited selectively to impaired mitochondria and promotes their autophagy. J Cell Biol 2008, 183:795-803

52. Okatsu K, Saisho K, Shimanuki M, Nakada K, Shitara H, Sou YS, Kimura M, Sato S, Hattori N, Komatsu M, Tanaka K, Matsuda N: p62/SQSTM1 cooperates with Parkin for perinuclear clustering of depolarized mitochondria. Genes Cells 2010, 15:887-900

53. Twig G, Shirihai OS: The interplay between mitochondrial dynamics and mitophagy. Antioxid Redox Signal 2011, 14:1939-1951

54. Ishihara N, Nomura M, Jofuku A, Kato H, Suzuki SO, Masuda K, Otera H, Nakanishi Y, Nonaka I, Goto Y, Taguchi N, Morinaga H, Maeda M, Takayanagi R, Yokota S, Mihara K: Mitochondrial fission factor Drp1 is essential for embryonic development and synapse formation in mice. Nat Cell Biol 2009, 11:958-966

55. Perdiz D, Lorin S, Leroy-Gori I, Pous C: Stress-induced hyperacetylation of microtubule enhances mitochondrial fission and modulates the phosphorylation of Drp1 at 616Ser. Cell Signal 2017, 39:32-43

56. Lin C, Kang J, Zheng R: Vitamin K3 triggers human leukemia cell death through hydrogen peroxide generation and histone hyperacetylation. Pharmazie 2005, 60:765-771

57. Whitworth AJ, Pallanck LJ: PINK1/Parkin mitophagy and neurodegeneration-what do we really know in vivo? Curr Opin Genet Dev 2017, 44:47-53

58. Wong YC, Holzbaur EL: Optineurin is an autophagy receptor for damaged mitochondria in parkin-mediated mitophagy that is disrupted by an ALS-linked mutation. Proc Natl Acad Sci U S A 2014, 111:E4439-E4448

59. Yamamoto A, Tagawa Y, Yoshimori T, Moriyama Y, Masaki R, Tashiro Y: Bafilomycin A1 prevents maturation of autophagic vacuoles by inhibiting fusion between autophagosomes and lysosomes in rat hepatoma cell line, H-4-II-E cells. Cell Struct Funct 1998, 23: $33-42$

60. Vives-Bauza C, Zhou C, Huang Y, Cui M, de Vries RL, Kim J, May J, Tocilescu MA, Liu W, Ko HS, Magrané J, Moore DJ, Dawson VL, Grailhe R, Dawson TM, Li C, Tieu K, Przedborski S: PINK1-dependent recruitment of Parkin to mitochondria in mitophagy. Proc Natl Acad Sci U S A 2010, 107:378-383

61. Rakovic A, Grunewald A, Seibler P, Ramirez A, Kock N, Orolicki S, Lohmann K, Klein C: Effect of endogenous mutant and wild-type PINK1 on Parkin in fibroblasts from Parkinson disease patients. Hum Mol Genet 2010, 19:3124-3137

62. Shiba-Fukushima K, Imai Y, Yoshida S, Ishihama Y, Kanao T, Sato S, Hattori N: PINK1-mediated phosphorylation of the Parkin ubiquitin-like domain primes mitochondrial translocation of Parkin and regulates mitophagy. Sci Rep 2012, 2:1002

63. Springer W, Kahle PJ: Regulation of PINK1-Parkin-mediated mitophagy. Autophagy 2011, 7:266-278

64. Price FW Jr, Price MO: Evolution of endothelial keratoplasty. Cornea 2013, 32 Suppl 1:S28-S32 
65. Mendl N, Occhipinti A, Muller M, Wild P, Dikic I, Reichert AS: Mitophagy in yeast is independent of mitochondrial fission and requires the stress response gene WHI2. J Cell Sci 2011, 124: $1339-1350$

66. Bernhardt D, Muller M, Reichert AS, Osiewacz HD: Simultaneous impairment of mitochondrial fission and fusion reduces mitophagy and shortens replicative lifespan. Sci Rep 2015, 5:7885

67. Song M, Mihara K, Chen Y, Scorrano L, Dorn GW 2nd: Mitochondrial fission and fusion factors reciprocally orchestrate mitophagic culling in mouse hearts and cultured fibroblasts. Cell Metab 2015, 21:273-286

68. Yamashita SI, Jin X, Furukawa K, Hamasaki M, Nezu A, Otera H, Saigusa T, Yoshimori T, Sakai Y, Mihara K, Kanki T: Mitochondrial division occurs concurrently with autophagosome formation but independently of Drp1 during mitophagy. J Cell Biol 2016, 215:649-665

69. Dagda RK, Cherra SJ 3rd, Kulich SM, Tandon A, Park D, Chu CT: Loss of PINK1 function promotes mitophagy through effects on oxidative stress and mitochondrial fission. J Biol Chem 2009, 284: 13843-13855

70. Wood-Kaczmar A, Gandhi S, Yao Z, Abramov AY, Miljan EA, Keen G, Stanyer L, Hargreaves I, Klupsch K, Deas E, Downward J, Mansfield L, Jat P, Taylor J, Heales S, Duchen MR, Latchman D, Tabrizi SJ, Wood NW: PINK1 is necessary for long term survival and mitochondrial function in human dopaminergic neurons. PLoS One 2008, 3:e2455

71. Wang D, Qian L, Xiong H, Liu J, Neckameyer WS, Oldham S, Xia K, Wang J, Bodmer R, Zhang Z: Antioxidants protect PINK1dependent dopaminergic neurons in Drosophila. Proc Natl Acad Sci U S A 2006, 103:13520-13525

72. Valente EM, Abou-Sleiman PM, Caputo V, Muqit MM, Harvey K, Gispert S, Ali Z, Del Turco D, Bentivoglio AR, Healy DG, Albanese A, Nussbaum R, Gonzalez-Maldonado R, Deller T, Salvi S, Cortelli P, Gilks WP, Latchman DS, Harvey RJ, Dallapiccola B, Auburger G, Wood NW: Hereditary early-onset Parkinson's disease caused by mutations in PINK1. Science 2004, 304:1158-1160

73. Kubli DA, Cortez MQ, Moyzis AG, Najor RH, Lee Y, Gustafsson AB: PINK1 is dispensable for mitochondrial recruitment of Parkin and activation of mitophagy in cardiac myocytes. PLoS One 2015, 10:e130707

74. Park J, Lee SB, Lee S, Kim Y, Song S, Kim S, Bae E, Kim J, Shong M, Kim JM, Chung J: Mitochondrial dysfunction in Drosophila PINK1 mutants is complemented by parkin. Nature 2006 , 441:1157-1161

75. Fischer TD, Hylin MJ, Zhao J, Moore AN, Waxham MN, Dash PK: Altered mitochondrial dynamics and TBI pathophysiology. Front Syst Neurosci 2016, 10:29

76. Chaugule VK, Burchell L, Barber KR, Sidhu A, Leslie SJ, Shaw GS, Walden H: Autoregulation of Parkin activity through its ubiquitin-like domain. EMBO J 2011, 30:2853-2867

77. Tang MY, Vranas M, Krahn AI, Pundlik S, Trempe JF, Fon EA: Structure-guided mutagenesis reveals a hierarchical mechanism of Parkin activation. Nat Commun 2017, 8:14697

78. Lazarou M, Jin SM, Kane LA, Youle RJ: Role of PINK1 binding to the TOM complex and alternate intracellular membranes in recruitment and activation of the E3 ligase Parkin. Dev Cell 2012, 22: 320-333

79. Engler C, Kelliher C, Spitze AR, Speck CL, Eberhart CG, Jun AS Unfolded protein response in fuchs endothelial corneal dystrophy: a unifying pathogenic pathway? Am J Ophthalmol 2010, 149: 194-202.e2

80. Elhalis H, Azizi B, Jurkunas UV: Fuchs endothelial corneal dystrophy. Ocul Surf 2010, 8:173-184

81. Okumura N, Hashimoto K, Kitahara M, Okuda H, Ueda E, Watanabe K, Nakahara M, Sato T, Kinoshita S, Tourtas T, SchlotzerSchrehardt U, Kruse F, Koizumi N: Activation of TGF-beta signaling induces cell death via the unfolded protein response in Fuchs endothelial corneal dystrophy. Sci Rep 2017, 7:6801

82. Wang Y, Nartiss Y, Steipe B, McQuibban GA, Kim PK: ROSinduced mitochondrial depolarization initiates PARK2/PARKINdependent mitochondrial degradation by autophagy. Autophagy 2012, 8:1462-1476

83. Kim EC, Meng H, Jun AS: N-Acetylcysteine increases corneal endothelial cell survival in a mouse model of Fuchs endothelial corneal dystrophy. Exp Eye Res 2014, 127:20-25 